\title{
POPULATION STUDIES. XIII. A NEW ANALYSIS OF THE BIDELMAN-MACCONNELL "WEAK-METAL" STARS-CONFIRMATION OF METAL-POOR STARS IN THE THICK DISK OF THE GALAXY
}

\author{
Timothy C. Beers ${ }^{1}$, John E. Norris ${ }^{2}$, Vinicius M. Placco ${ }^{3}$, Young Sun LeE ${ }^{4}$, Silvia Rossi ${ }^{5}$, \\ Daniela Carollo ${ }^{6}$, And Thomas Masseron ${ }^{7}$ \\ ${ }^{1}$ Department of Physics and JINA Center for the Evolution of the Elements, University of Notre Dame, Notre Dame, IN 46556, USA; tbeers@nd.edu \\ ${ }^{2}$ Research School of Astronomy and Astrophysics, The Australian National University, Mount Stromlo Observatory, \\ Cotter Road, Weston, ACT 2611, Australia; jen@mso.anu.edu.au \\ ${ }^{3}$ Gemini Observatory, Hilo, HI 96720, USA; vplacco@gemini.edu \\ ${ }^{4}$ Department of Astronomy and Space Science, Chungnam National University, Daejeon 305-764, Republic of Korea; youngsun@cnu.ac.kr \\ ${ }^{5}$ Instituto de Astronomia, Geofísica e Ciências Atmosféricas, Departamento de Astronomia, Universidade de São Paulo, \\ Rua do Matão 1226, 05508-900 São Paulo, Brazil; rossi@ astro.iag.usp.br \\ ${ }^{6}$ Department of Physics and Astronomy, Astronomy, Astrophysics and Astrophotonic Research Center, \\ Macquarie University, North Ryde 2019, NSW, Australia; daniela.carollo@mq.edu.au \\ ${ }^{7}$ Institute of Astronomy, University of Cambridge, CB3 0HA, UK; tpm40@ ast.cam.ac.uk \\ Received 2014 June 10; accepted 2014 August 10; published 2014 September 24
}

\begin{abstract}
A new set of very high signal-to-noise $(\mathrm{S} / \mathrm{N}>100 / 1)$, medium-resolution $(R \sim 3000)$ optical spectra have been obtained for 302 of the candidate "weak-metal" stars selected by Bidelman \& MacConnell. We use these data to calibrate the recently developed generalization of the Sloan Extension for Galactic Exploration and Understanding and Exploration (SEGUE) Stellar Parameter Pipeline, and obtain estimates of the atmospheric parameters $\left(T_{\text {eff }}\right.$, $\log g$, and $[\mathrm{Fe} / \mathrm{H}])$ for these non-Sloan Digital Sky Survey/SEGUE data; we also obtain estimates of [C/Fe]. The new abundance measurements are shown to be consistent with available high-resolution spectroscopic determinations, and represent a substantial improvement over the accuracies obtained from the previous photometric estimates reported in Paper I of this series. The apparent offset in the photometric abundances of the giants in this sample noted by several authors is confirmed by our new spectroscopy; no such effect is found for the dwarfs. The presence of a metal-weak thick-disk (MWTD) population is clearly supported by these new abundance data. Some $25 \%$ of the stars with metallicities $-1.8<[\mathrm{Fe} / \mathrm{H}] \leqslant-0.8$ exhibit orbital eccentricities $e<0.4$, yet are clearly separated from members of the inner-halo population with similar metallicities by their location in a Lindblad energy versus angular momentum diagram. A comparison is made with recent results for a similar-size sample of Radial Velocity Experiment stars from Ruchti et al. We conclude, based on both of these samples, that the MWTD is real, and must be accounted for in discussions of the formation and evolution of the disk system of the Milky Way.
\end{abstract}

Key words: Galaxy: kinematics and dynamics - Galaxy: stellar content - Galaxy: structure - stars: abundances stars: Population II

Online-only material: color figures, machine-readable tables

\section{INTRODUCTION}

\subsection{Historical Overview}

In the first paper of this series, Norris et al. (1985, hereafter NBP, Paper I) presented David Dunlap Observatory (DDO) photometric estimates of metallicity, spectroscopic measurements of radial velocities (RVs), and photometric classifications for a sample of 309 non-kinematically selected stars taken from the list of "weak-metal" candidates originally identified by Bidelman \& MacConnell (1973). Based on these data, and supplemented with proper motions that were available at the time, NBP obtained space motions and estimates of orbital eccentricities for a subset of this sample. Inspection of this distribution led these authors to conclude that there exists a substantial number of low-metallicity stars on low-eccentricity orbits $(e<0.4)$, which they found difficult to reconcile with the classical rapid collapse model for the formation of the Galaxy put forward by Eggen, Lynden-Bell, \& Sandage (1962, hereafter ELS). NBP suggested instead that the low-metallicity, low-eccentricity stars belong to a population that is "(1) ... intermediate in its motion perpendicular to the Galactic plane between that of the thin disk and that of metal-deficient objects of extreme eccentricity, and (2) that the velocity dispersion of this group of stars is consistent with its belonging to the thick-disk population described by Gilmore (1984)" (NBP, p. 488). This suggested population has become known as the metal-weak thick disk (MWTD; e.g., Morrison et al. 1990; Beers \& Sommer-Larsen 1995).

Attempts to confirm or refute the existence of a MWTD population have led to numerous (and ever more-detailed) studies over the past two decades. Morrison et al. (1990) provided additional support for the MWTD, based on a (slightly revised) DDO photometric abundance scale, and the kinematics of a low-latitude sample of giants selected to test for a separation of halo-like and disk-like objects. However, the conclusions of both of these efforts were called into question by subsequent work. In the case of NBP, Anthony-Twarog \& Twarog (1994) obtained an improved calibration of the DDO abundance estimates for their sample giants, and concluded that there existed an offset of about 0.5 dex for giants of intermediate metallicity (around $[\mathrm{Fe} / \mathrm{H}]=-1.2$ ). As a result, these authors suggested that the numbers of stars with disk-like motions and metallicities $[\mathrm{Fe} / \mathrm{H}]<-1.0$ in the work of NBP had been substantially overestimated, compromising the claim for a MWTD population. Ryan \& Lambert (1995) sought to resolve these discrepancies 
by obtaining high-resolution spectroscopic abundance determinations of some 30 giants in these two samples with claimed photometric abundance estimates $[\mathrm{Fe} / \mathrm{H}]<-1.0$. The results of their study indicated that many, but not all, of the giants in the NBP and the Morrison et al. (1990) sample possessed higher metallicities than had been inferred from the DDO photometry. As a consequence, they argued that, although a MWTD may indeed exist, its contribution to the populations of stars within $1 \mathrm{kpc}$ of the Galactic disk had likely been overestimated by previous work.

Other observational efforts have addressed the problem of the existence of a MWTD component in the Galaxy. For instance, Beers \& Sommer-Larsen (1995) argued from their sample of non-kinematically selected stars that a surprisingly large fraction of the metal-poor stars $(>30 \%$ of stars with $[\mathrm{Fe} / \mathrm{H}]<-1.5$, rising to $60 \%$ for stars with $-1.6 \leqslant$ $[\mathrm{Fe} / \mathrm{H}] \leqslant-1.0)$ in the solar neighborhood might be associated with a MWTD component. Chiba \& Yoshii (1998) used highquality proper motions from the Hipparcos satellite for a much smaller sample of red giants and RR Lyraes to argue that, while a MWTD appeared present, the fractions of stars at low metallicity associated with it were substantially smaller, roughly $10 \%$ for stars in the interval $-1.6 \leqslant[\mathrm{Fe} / \mathrm{H}] \leqslant-1.0$. Martin \& Morrison (1998) considered the space motions of nearby RR Lyrae stars with well-determined kinematics, and concluded that a MWTD existed in their sample (including stars with metallicities as low as $[\mathrm{Fe} / \mathrm{H}] \sim-2.0)$, similar to previous results for the sample of RR Lyraes examined by Layden (1995).

Chiba \& Beers (2000) performed a detailed analysis of a large sample of non-kinematically selected stars with available (medium-resolution) spectroscopic abundances, RVs, and (for roughly half of their sample) proper motions from the assembly of Beers et al. (2000). These authors concluded that the fraction of likely MWTD stars in the solar neighborhood with $-1.7<$ $[\mathrm{Fe} / \mathrm{H}] \leqslant-1.0$ was on the order of $30 \%$, falling to on the order of $10 \%$ for stars with $-2.2<[\mathrm{Fe} / \mathrm{H}] \leqslant-1.7$. Beers et al. (2002) analyzed a sample of candidate low-metallicity giants located close to the Galactic plane from the Luminous Stars Extension (LSE) survey of Drilling \& Bergeron (1995). Their Monte Carlo experiments on the distribution of orbital eccentricities of this sample suggested that the fraction of MWTD stars with [Fe/ $\mathrm{H}]<-1.0$ might actually be as high as $40 \%$, and that it may remain as high as $30 \%$ for stars with $[\mathrm{Fe} / \mathrm{H}]<-1.6$. Beers et al. reasoned that the origin of this discrepancy with respect to the work of Chiba \& Beers (2000) came from the selection criteria employed by most surveys for low-metallicity stars, which understandably concentrated on regions of the Galaxy with latitudes above $|b|=30^{\circ}$.

Arifyanto et al. (2005) re-analyzed the kinematically selected sample of Carney et al. (1994), using Hipparcos-based parallaxes and (where available) Hipparcos and Tycho-2 proper motions, and applying corrections to the Carney et al. photometric distance estimates based on the overlap of the two samples. Their analysis also indicated the presence of a MWTD component, with a local stellar fraction smaller than that claimed by Chiba \& Beers (2000) in the metallicity interval $-1.7<$ $[\mathrm{Fe} / \mathrm{H}] \leqslant-1.0(18 \%$ versus $30 \%)$. They interpreted the origin of the MWTD in terms of the debris of a "shredded satellite," similar to the argument of Gilmore et al. (2002). It is notable that Gilmore et al. (2002) concluded, based on an analysis of their spectroscopic survey of some $2000 \mathrm{~F} / \mathrm{G}$ stars located $0.5-5 \mathrm{kpc}$ above the Galactic plane, that the stars they proposed to originate in a shredded satellite exhibited a large rotational velocity lag with respect to the thin/thick disk, on the order of $100 \mathrm{~km} \mathrm{~s}^{-1}$. Previous analyses for potential MWTD stars generally did not consider stars with such a large lag as likely candidate disklike stars. Large lags for accreted MWTD stars may in fact be expected, as argued by Villalobos \& Helmi (2009). Clearly, care must be exercised in the selection of potential MWTD stars before attempting to discern their kinematic and chemical properties.

\subsection{The Nature and Role of the MWTD in the Context of the Milky Way}

The preponderance of evidence acquired prior to 2009 suggested that a MWTD component exists, although doubts remained as to its level of contribution to the numbers of metalpoor stars in the solar neighborhood, as well as regarding its detailed kinematical behavior and relationship to the canonical thick-disk component, and to the halo. In the period since 2009, a substantial volume of work has been carried out, making use of large samples of stars with medium-resolution $(R \sim 2000)$ spectroscopy obtained from a variety of surveys, in particular the Sloan Digital Sky Survey (SDSS; York et al. 2000), and its Galactic extensions, the Sloan Extension for Galactic Exploration and Understanding and Exploration-1 (SEGUE-1; Yanny et al. 2009) and SEGUE-2 (C. Rockosi et al., in preparation), as well as higher-resolution $(R \sim 7500)$ data from the Radial Velocity Experiment (RAVE; Steinmetz et al. 2006), and other sources. A partial list of these works includes Carollo et al. (2010), Ruchti et al. (2010, 2011), Kordopatis et al. (2011, 2013a, 2013b), Lee et al. (2011), Bovy et al. (2012a, 2012b, 2012c), Carrell et al. (2012), Cheng et al. (2012a, 2012b), Pasetto et al. (2012), Adibekyan et al. (2013), Boeche et al. (2013a, 2013b), Haywood et al. (2013), Jayaraman et al. (2013), Bensby et al. (2014), and Minchev et al. (2014).

During this period, our appreciation of the complexity of the halo has also increased. Carollo et al. (2007, 2010) and Beers et al. (2012) have presented the case that this system is well described in terms of an inner-halo and outer-halo population-terms that we shall use in what follows. ${ }^{9}$ Additional evidence supporting the existence of (at least) a dual halo has come from recognition that the frequency of carbon-enhanced metal-poor (CEMP) stars that can be kinematically associated with the outer-halo component is roughly twice that of the innerhalo component (Carollo et al. 2012), analysis of the metallicity distribution function (MDF), in combination with the motions, of local halo stars by An et al. (2014), the apparent preference for stars of the CEMP-s sub-class (those exhibiting $s$-processelement overabundances) to be associated with the inner-halo component, while stars of the CEMP-no sub-class (those exhibiting no neutron-capture overabundances) are more likely associated with the outer-halo component (Carollo et al. 2014), and analysis of the in-situ change of the halo system MDF with distance for a large sample of F-turnoff stars from SDSS (Allende Prieto et al. 2014).

Furthermore, Morrison et al. (2009) have used a sample of some 250 stars with very well-determined kinematical properties to argue for the presence of a new component of the local halo, with an axial ratio $c / a \sim 0.2$ (similar in flattening to the thick disk) and populated by stars with $-1.5<[\mathrm{Fe} / \mathrm{H}]<-1.0$, which is, however, not rotationally supported. The potential

\footnotetext{
9 We note for completeness that an alternative view has been expressed by Schönrich et al. (2011).
} 
confusion of such stars with MWTD candidates is obvious, due to their proximity to the Galactic plane.

These works have raised new and interesting questions concerning the nature of the formation and evolution of both the disk and halo systems. In the discussion of the MWTD, there seems to be no consensus yet as to what the inter-relationships are between it, the canonical thick disk, and the thin disk. Are they independent and discrete sub-systems? What have been the roles (if any) of major mergers, the formation of the thin disk from an early thick disk, the heating of a pre-existing thin stellar disk by minor mergers, and/or efficient radial migration of stars in the plane of the disk? We call the reader's attention to the insightful discussion by Haywood et al. (2013) of the apparently disparate results concerning the nature of the thick/thin disks arising from several recent studies. The issues being considered are clearly complex, even in the face of high-quality data, and subtleties of the approaches used and conclusions reached ensure that we have not yet arrived at a widely accepted view.

\subsection{Scope of Present Investigation}

The focus of this paper is considerably narrower. Here, we re-investigate the original sample of Bidelman \& MacConnell (1973) discussed by NBP, in order to resolve whether or not it includes substantial numbers of stars that could be considered members of the MWTD population. New high signal-to-noise $(\mathrm{S} / \mathrm{N})$ medium-resolution $(R \sim 3000)$ spectroscopy has been obtained for some 300 stars of the NBP sample. Roughly onethird of this sample now has available high-resolution spectroscopic determinations of $[\mathrm{Fe} / \mathrm{H}]$ (and other physical parameters) from the literature, which we employ to carry out a calibration of the physical parameter estimates obtained by a "non-SEGUE" version of the SEGUE Stellar Parameter Pipeline (SSPP; originally described by Lee et al. 2008a), and referred to as the n-SSPP. We also report estimates of "carbonicity," [C/Fe], calibrated with respect to some 50 of the NBP stars with previous high-resolution spectroscopic determinations of this ratio reported in the literature, supplemented by a number of additional stars with available high-resolution determinations. The results of this calibration effort will be used in a number of future investigations based on non-SEGUE spectroscopic data. We then combine available RVs, accurate proper motions from the Hipparcos and Tycho-II catalogs, and the newly refined spectroscopic estimates of $[\mathrm{Fe} / \mathrm{H}]$ to consider the presence of a MWTD in this sample. The resulting determinations of kinematic estimates represent a substantial improvement in the space velocities derived by NBP, sharpening the picture of the stellar populations obtained from these data, and confirming the suggestion of NBP that the ELS paradigm is an oversimplification of the manner in which the Milky Way formed.

This paper is outlined as follows. Our new observations are described in Section 2, where we also discuss the determination of RVs and spectroscopic line-strength indices for the program stars. Section 3 describes the techniques used to obtain estimates of the stellar atmospheric parameters $\left(T_{\text {eff }}, \log g,[\mathrm{Fe} / \mathrm{H}]\right)$, as well as $[\mathrm{C} / \mathrm{Fe}]$, for these stars. A comparison of the newly derived metallicity estimates with available high-resolution abundance results drawn from the recent literature and with the original DDO photometry-based estimates of NBP is presented in this section as well. We then make use of these comparisons to carry out a calibration of the n-SSPP. Distance estimates and proper motions for our sample stars are described in Section 4. In Section 5, we use these data to perform a new kinematic analysis of the NBP sample, and compare with the kinematics derived from a similar sample of RAVE stars described by Ruchti et al. (2011). A brief discussion of the implications of our new results is presented in Section 6.

\section{SPECTROSCOPIC OBSERVATIONS, AVAILABLE PHOTOMETRIC MEASUREMENTS, AND DERIVATION OF RADIAL VELOCITIES AND LINE INDICES}

\subsection{Details of Spectroscopic Observations}

During several observing runs conducted in 1996 January, 1996 December, and 1997 June, optical spectra for a total of 302 stars from the subsample of 309 Bidelman \& Maconnell weak-metal candidates studied by NBP (hereafter referred to as the B\&M sample) were obtained with the Siding Spring Observatory (SSO) $2.3 \mathrm{~m}$ telescope, using the Double Beam Spectrograph. These spectra covered the wavelength interval $3800 \AA \leqslant \lambda \leqslant 4500 \AA$, with a resolving power of $R \sim 3000$, similar to that obtained during the course of previous work on follow-up spectroscopy by Norris et al. (1999) of metalpoor candidates selected from the HK survey of Beers et al. $(1985,1992)$. However, because the stars in the current program are, in general, quite bright $(6.7<V<11.0)$, high-quality spectra (with $\mathrm{S} / \mathrm{N}>100$ per resolution element) could be obtained in reasonably short integration times. A total of 383 spectra were obtained, including a number of stars with repeated measurements. These spectra were reduced using standard procedures for flat-fielding, extraction, and wavelength calibration (based on arc-lamp exposures taken immediately before or after each science spectrum), using the FIGARO software (Shortridge 1993) and ancillary FORTRAN routines. No attempt to spectrophotometrically calibrate the spectra was made.

Figure 1 provides examples of the medium-resolution spectra obtained. Also shown on the plots are the stellar atmospheric parameters, determined using the methods described below (Section 3). The left column of panels shows two examples of stars classified as dwarfs by NBP (using taxonomy based on DDO photometry), as well as by our own spectroscopic analysis. The upper left panel is the spectrum of a metal-poor dwarf (BM-060 = CD-48:1741), while the lower left panel is the spectrum of a dwarf with solar metallicity (BM-047 = BD-13:959). The right column of panels provides examples of spectra for stars classified as giants (by both NBP and the present work). The upper right panel is the spectrum of a metalpoor giant (BM-120 = HD 84903), while the lower right panel is the spectrum for a giant with metallicity slightly above solar (BM-072 = HD 40361).

\subsection{Broadband Photometry and Reddening Estimation}

The NBP study provided broadband $V$ magnitudes and $B-V$ colors for the majority of our program objects. We have checked the SIMBAD database for consistency with other measurements, and in a few cases replaced the values listed by NBP with what we judged to be an improved set of information. In some cases, photometry was not available from NBP. For these stars, we adopted values provided in the SIMBAD database. The results are listed in Table 1. In this table, Column 1 lists the star names used by NBP, in the format BM-XXX, while Column 2 lists a more commonly used name for the star (e.g., BD, CD, HD, etc.). Columns 3 and 4 are the Galactic longitude and latitude in decimal degrees, respectively. Columns 5 and 6 list 

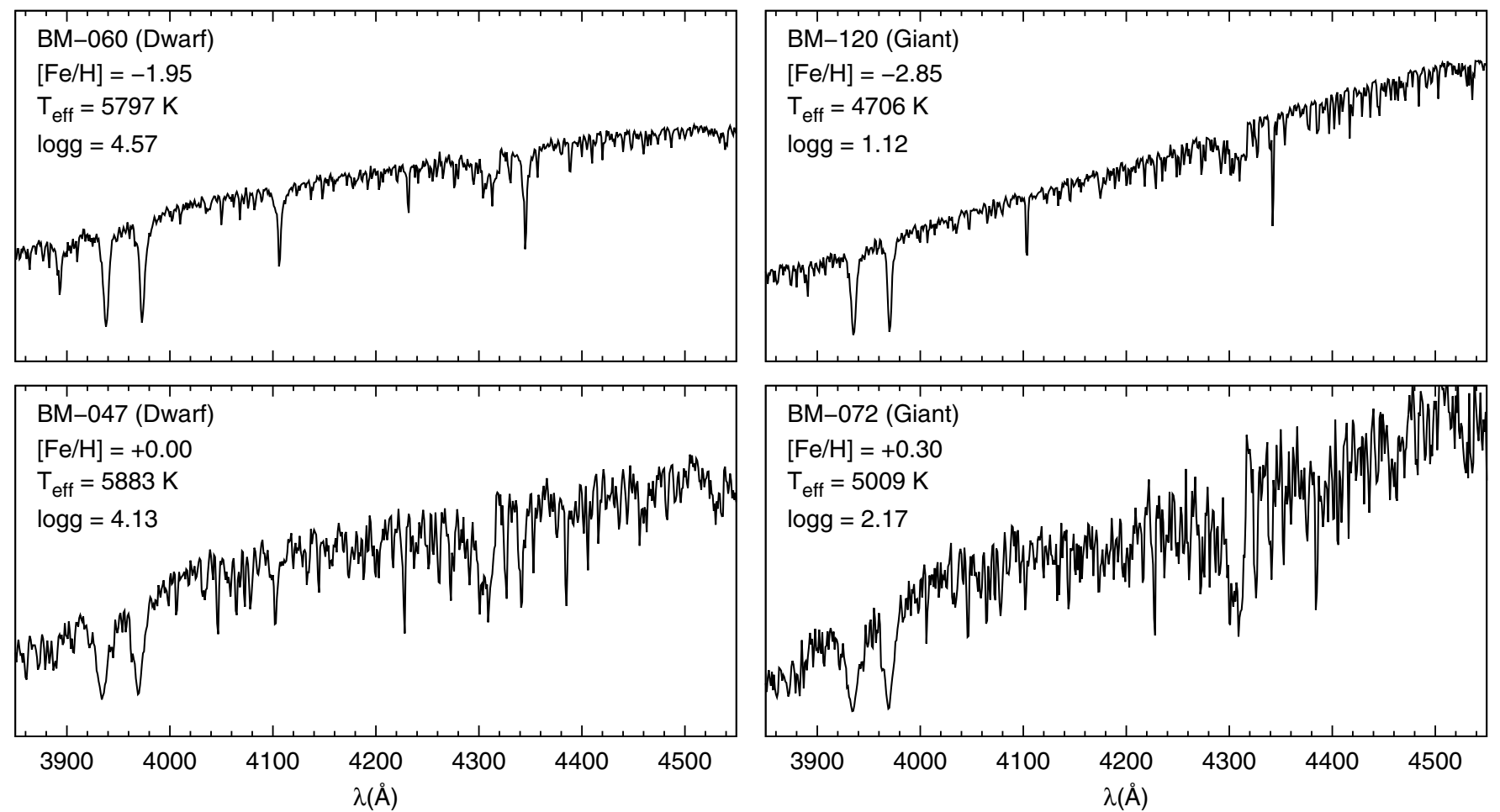

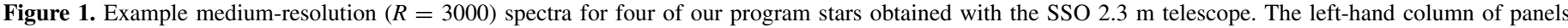

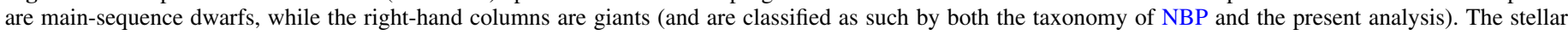
atmospheric parameters from our analysis are indicated in the legend of each spectrum.

Table 1

Photometric Information and Adopted Reddening

$\left.\begin{array}{lcccccccccc}\hline \hline \text { BM Name } & \text { Star Name } & \begin{array}{c}\text { LON } \\ \left({ }^{\circ}\right)\end{array} & \begin{array}{c}\text { LAT } \\ \left({ }^{\circ}\right)\end{array} & \begin{array}{c}V \\ (\mathrm{mag})\end{array} & \begin{array}{c}B-V \\ (\mathrm{mag})\end{array} & \begin{array}{c}J \\ (\mathrm{mag})\end{array} & \begin{array}{c}J-K \\ (\mathrm{mag})\end{array} & \begin{array}{c}E(B-V)_{S} \\ (\mathrm{mag})\end{array} & \begin{array}{c}E(B-V)_{A} \\ (\mathrm{mag})\end{array} & \begin{array}{c}E(B-V)_{F} \\ (\mathrm{mag})\end{array} \\ (1) & (2) & (3) & (4) & (5) & (6) & (7) & (8) & 0.034 & 0.03 \\ (11)\end{array}\right)$

(This table is available in its entirety in a machine-readable form in the online journal. A portion is shown here for guidance regarding its form and content.)

the adopted $V$ magnitude and $B-V$ colors, respectively. NearIR $J H K$ photometry is available for the bulk of our sample, based on results from the Two Micron All Sky Survey (2MASS) catalog (Skrutskie et al. 2006). The $J$ magnitude and $J-K$ colors reported by $2 \mathrm{MASS}$ for stars without flags indicating potential problems in the listed values are given in Columns 7 and 8 , respectively.

In order to obtain absorption- and reddening-corrected estimates of the magnitudes and colors, respectively, we initially adopt the Schlegel et al. (1998) estimates of reddening, listed in Column 9 of Table 1 . We have applied corrections to these estimates for objects with reddening greater than $E(B-V)_{S}=0.10$, as described by Beers et al. (2002). The corrected reddening estimates, $E(B-V)_{A}$, are listed in Column 10 of Table 1 . The final reddening estimates must be obtained in conjunction with the distance estimates, obtained as described below, in order to properly account for the amount of foreground reddening suffered by each star. The final estimated reddenings, $E(B-V)_{F}$, are listed in Column 11 . Note that about $20 \%$ of our sample stars are located at low Galactic latitudes, $|b|<10^{\circ}$, for which the reddening estimate along the line of sight to a star is unreliable. For these stars we simply set the reddening estimate to zero for the initial parameter analysis. ${ }^{10}$

\subsection{Measurement of Radial Velocities and Line Indices}

Our program stars, and the number of medium-resolution spectra obtained for each star, are listed in the first two columns of Table 2. RVs were measured for our program objects using

\footnotetext{
10 The n-SSPP employs multiple approaches, some of which use spectroscopic-only input information, which provides robustness in the parameter estimates in spite of spurious reddening estimates.
} 
Table 2

Radial Velocities, Line Indices, Atmospheric Parameters, and Type Assignments

\begin{tabular}{|c|c|c|c|c|c|c|c|c|c|c|c|c|c|c|c|c|c|c|c|}
\hline BM Name & $\begin{array}{l}N \\
\text { (2) }\end{array}$ & $\begin{array}{c}\mathrm{RV}_{M} \\
\left(\mathrm{~km} \mathrm{~s}^{-1}\right) \\
(3)\end{array}$ & $\begin{array}{c}\mathrm{RV}_{H} \\
\left(\mathrm{~km} \mathrm{~s}^{-1}\right) \\
(4)\end{array}$ & $\begin{array}{l}\mathrm{KP} \\
(\AA) \\
(5)\end{array}$ & $\begin{array}{l}\text { HP2 } \\
(\AA) \\
(6)\end{array}$ & $\begin{array}{l}\text { GP } \\
(\AA) \\
(7)\end{array}$ & $\begin{array}{l}\text { HG2 } \\
(\AA) \\
(8)\end{array}$ & $\begin{array}{c}\text { LACF } \\
(9)\end{array}$ & $\begin{array}{l}T_{\text {eff } S} \\
(\mathrm{~K}) \\
(10)\end{array}$ & $\begin{array}{c}\log g_{S} \\
(\mathrm{cgs}) \\
(11)\end{array}$ & $\begin{array}{c}{[\mathrm{Fe} / \mathrm{H}]_{S}} \\
(12)\end{array}$ & $\begin{array}{l}T_{\text {eff } H} \\
(\mathrm{~K}) \\
(13)\end{array}$ & $\begin{array}{c}\log g_{H} \\
(\operatorname{cgs}) \\
(14)\end{array}$ & $\begin{array}{c}{[\mathrm{Fe} / \mathrm{H}]_{H}} \\
(15)\end{array}$ & $\begin{array}{c}T_{\text {eff } C} \\
(\mathrm{~K}) \\
(16)\end{array}$ & $\begin{array}{c}\log g_{C} \\
(\mathrm{cgs}) \\
(17)\end{array}$ & $\begin{array}{c}{[\mathrm{Fe} / \mathrm{H}]_{C}} \\
(18)\end{array}$ & $\begin{array}{c}{[\mathrm{Fe} / \mathrm{H}]_{N}} \\
(19)\end{array}$ & $\begin{array}{c}\text { TYPE }_{N / S} \\
\text { (20) }\end{array}$ \\
\hline BM-001 & 1 & 45 & $\ldots$ & 7.90 & 2.85 & 3.39 & 2.53 & 0.204 & 6007 & 3.75 & -0.80 & & $\cdots$ & & 6001 & 4.11 & -0.56 & -0.44 & $\mathrm{D} / \mathrm{D}$ \\
\hline BM-002 & 2 & -48 & -61 & 6.37 & 1.06 & 1.66 & 1.25 & -0.347 & 5065 & 1.15 & -2.46 & 4901 & 1.44 & -2.30 & 4894 & 0.90 & -2.60 & -2.43 & $\mathrm{RG} / \mathrm{G}$ \\
\hline BM-003 & 1 & -32 & -48 & 6.39 & 3.51 & 1.64 & 3.45 & -0.233 & 6105 & 3.58 & -1.27 & 6004 & 4.06 & -1.21 & 6117 & 3.90 & -1.14 & -0.82 & $\mathrm{D} / \mathrm{TO}$ \\
\hline BM-004 & 1 & 29 & $\ldots$ & 6.70 & 4.19 & 2.24 & 4.06 & 0.027 & 6355 & 3.75 & -0.66 & & $\ldots$ & $\ldots$ & 6411 & 4.11 & -0.39 & -0.42 & $\mathrm{D} / \mathrm{D}$ \\
\hline BM-005 & 1 & -68 & -66 & 4.86 & 1.62 & 2.60 & 1.14 & -0.662 & 5266 & 1.70 & -2.42 & 4935 & 1.98 & -2.73 & 5130 & 1.58 & -2.55 & -2.45 & $\mathrm{RG} / \mathrm{G}$ \\
\hline BM-006 & 3 & 9 & 8 & 10.01 & 0.84 & 5.65 & 1.15 & 0.472 & 5123 & 2.39 & -1.19 & 5116 & 2.30 & -0.93 & 4962 & 2.43 & -1.04 & -1.44 & $\mathrm{RG} / \mathrm{G}$ \\
\hline BM-007 & 1 & 122 & 119 & 9.84 & 0.71 & 5.47 & 0.87 & 0.605 & 5285 & 2.69 & -0.69 & 5079 & 2.79 & -0.83 & 5152 & 2.80 & -0.42 & -1.55 & $\mathrm{RG} / \mathrm{G}$ \\
\hline BM-008 & 1 & 47 & $\ldots$ & 8.52 & 2.00 & 4.56 & 1.88 & 0.439 & 5821 & 4.29 & -0.35 & 6006 & $\ldots$ & $\ldots$ & 5783 & 4.78 & 0.00 & -0.47 & $\mathrm{D} / \mathrm{D}$ \\
\hline BM-009 & 1 & 7 & -5 & 8.74 & 1.91 & 4.65 & 1.77 & 0.402 & 5693 & 4.37 & -0.82 & 5664 & 4.38 & -0.71 & 5632 & 4.88 & -0.58 & -0.78 & $\mathrm{D} / \mathrm{D}$ \\
\hline BM-010 & 1 & 28 & 23 & 6.38 & 4.28 & 1.98 & 4.12 & -0.037 & 6354 & 3.62 & -0.80 & $\ldots$ & $\ldots$ & $\ldots$ & 6409 & 3.95 & -0.56 & -0.38 & $\mathrm{D} / \mathrm{TO}$ \\
\hline
\end{tabular}

Notes. A ":" appended to Column 20 indicates a disagreement between the type assignment (based on photometry) in Paper I and that assigned based on the atmospheric parameters determined by the n-SSPP.

(This table is available in its entirety in a machine-readable form in the online journal. A portion is shown here for guidance regarding its form and content.) 

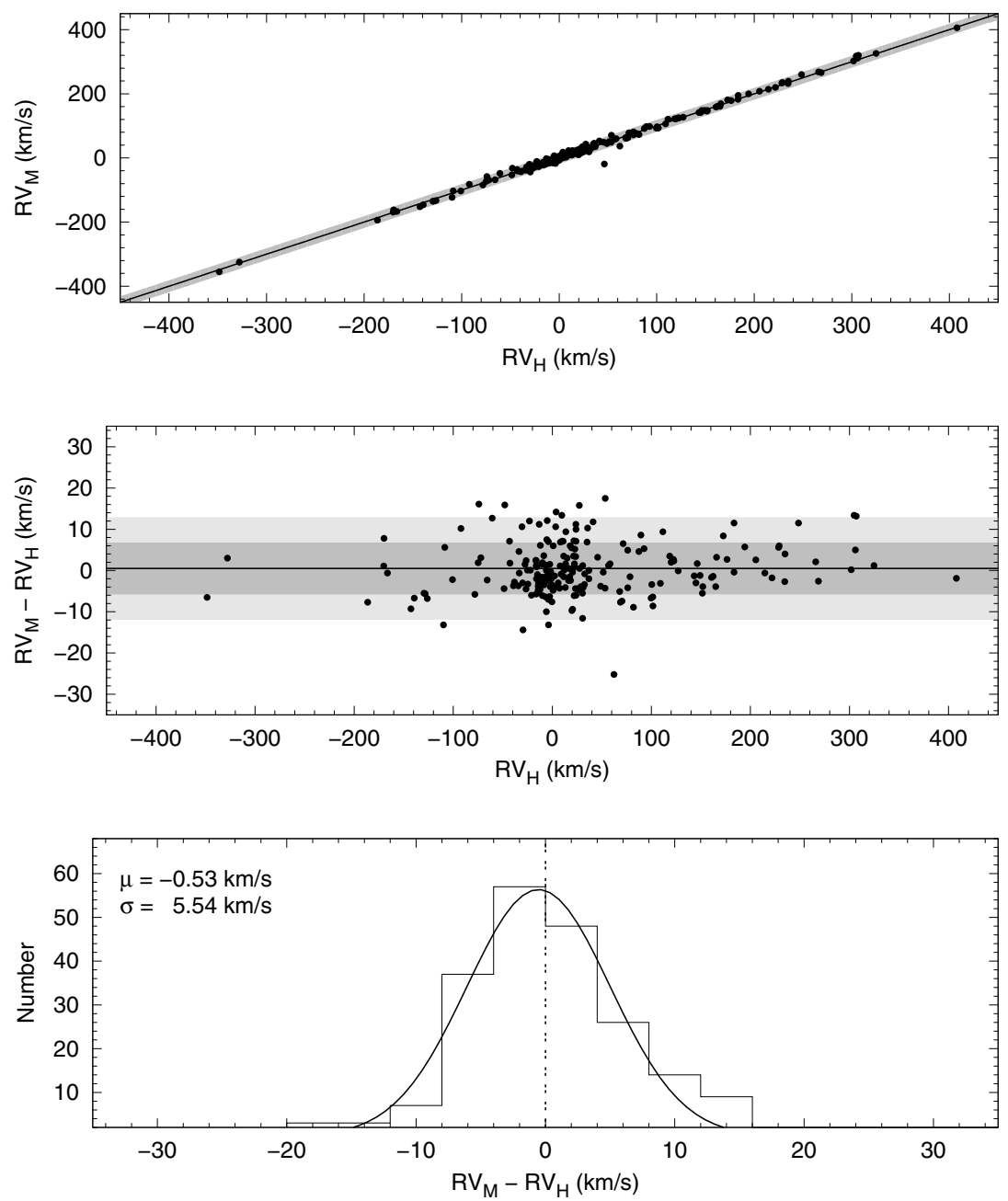

Figure 2. Upper panel: comparison between the radial velocities of our program stars, determined from the medium-resolution spectra using the techniques described by Beers et al. (1999), and those reported in the literature from analyses of high-resolution spectra. The solid line is the one-to-one line, and the shaded area represents a $3 \sigma$ interval around this line (where $\sigma$ represents the scatter in the residuals shown in the lower panel, $5.5 \mathrm{~km} \mathrm{~s}^{-1}$ ). Middle panel: residuals between the medium-resolution and high-resolution radial velocities, as a function of the high-resolution values. The horizontal solid line is the average of the residuals, while the darker and lighter shaded areas represent the $1 \sigma$ and $2 \sigma$ regions, respectively. Lower panel: histogram of the residuals in the radial-velocity determinations. The values of the mean offset and scatter are the parameters from the Gaussian fit shown.

the line-by-line and cross-correlation techniques described in detail by Beers et al. (1999), and references therein. The spectral resolution is similar to that obtained for the majority of the HK survey follow-up, so we expect that the measured RVs should be precise to the same level (or better, given the higher $\mathrm{S} / \mathrm{N}$ of our present spectra), on the order of $7-10 \mathrm{~km} \mathrm{~s}^{-1}(1 \sigma)$. Heliocentric RVs obtained from the medium-resolution spectra for our program stars, $\mathrm{RV}_{M}$, are listed in Column 3 of Table 2.

Roughly one-third of our program objects have had RVs determined from high-resolution spectroscopic studies (available results are provided in Column 4 of Table $2, \mathrm{RV}_{H}$ ). The upper two panels of Figure 2 compare $\mathrm{RV}_{M}$ with those obtained from the independent high-resolution observations. As can be appreciated from inspection of this figure, there is generally excellent agreement. A maximum likelihood fit to the residuals in $\mathrm{RV}$, as shown in the lower panel of Figure 2, indicates that the rms scatter is only on the order of $5.5 \mathrm{~km} \mathrm{~s}^{-1}$. Assuming that the (combined) high-resolution RVs from the literature have a precision on the order of $2.0 \mathrm{~km} \mathrm{~s}^{-1}$, the external errors in our medium-resolution RV determinations appear to be no worse than about $5 \mathrm{~km} \mathrm{~s}^{-1}$, slightly better than expected. This represents a factor of two improvement in the precision of the RVs reported by NBP.
Although we do not employ them for obtaining stellar metallicity estimates in the present paper, we have measured a set of spectral indices that have been used for this and other purposes in previous papers (e.g., alternative schemes for estimation of dereddened colors based on Balmer-line strengths, $[\mathrm{C} / \mathrm{Fe}]$ estimates based on the $\mathrm{CH} G$-band line index, etc.). Since these may prove useful in the future, and are very well measured in our high-S/N medium-resolution spectra, we describe their determination below.

For each star, the measured (geocentric) RVs were used to place a set of fixed bands for the derivation of line-strength indices, which are pseudo-equivalent widths of prominent spectral features. We employ a subset of the bands listed in Table 1 of Beers et al. (1999). ${ }^{11}$

Line indices for prominent spectral features for each of our program stars are reported in Columns 5-8 of Table 2. A number of our program stars had more than one spectrum obtained during the course of the follow-up observations. From a comparison of the stars with repeated measurements, we

11 The indices KP, HP2, GP, and HG2 measure the strength of the Ca II K line, hydrogen $\mathrm{H} \delta$, the $\mathrm{CH} G$-band, and hydrogen $\mathrm{H} \gamma$, respectively. A complete discussion of the choice of bands and the "band-switching" scheme used to determine the indices are provided in this reference as well. 
estimate that errors in the line indices on the order of $0.1 \AA$ are achieved. In order for a line-index measurement to be considered a detection, we require that the derived indices be above a minimum value of $0.25 \AA$. Indices that failed to reach this minimum value are indicated in the Table 2 as missing data.

In addition to the line-strength indices, we have measured an autocorrelation function index for each star, as described in detail in Beers et al. (1999), and references therein. We actually make use of the base-10 logarithm of this index, hence it is referred to as LACF (listed in Column 9 of Table 2). The LACF index quantifies the strength of the multitude of weak metallic lines that are present in each spectrum, and provides an additional indicator of the overall abundance. This index is of particular use for cooler and/or metal-rich stars, where the KP-index technique for inference of stellar metallicity approaches saturation.

\section{STELLAR ATMOSPHERIC PARAMETERS AND CARBON ABUNDANCE RATIOS}

Stellar atmospheric parameters for our program stars were determined using the n-SSPP, a modified version of the SSPP (see Lee et al. 2008a, 2008b, 2011; Allende Prieto et al. 2008; Smolinski et al. 2011 for a detailed description of the procedures used). The n-SSPP is a collection of routines for the analysis of non-SDSS/SEGUE data that employs both spectroscopic and photometric $\left(V_{0},(B-V)_{0}, J_{0}\right.$, and $\left.(J-K)_{0}\right)$ information as inputs, in order to make a series of estimates for each stellar parameter. ${ }^{12}$ Then, using $\chi^{2}$ minimization matching techniques within dense grids of synthetic spectra, and averaging with other techniques as available (depending on the wavelength range of the input spectra; see Table 5 of Lee et al. 2008a), the best set of values is adopted. For the SSPP, internal errors for the stellar parameters are $125 \mathrm{~K}$ for $T_{\text {eff }}, 0.25 \mathrm{dex}$ for $\log g$, and $0.20 \mathrm{dex}$ for $[\mathrm{Fe} / \mathrm{H}]$; external errors are of a similar size. We might expect the external errors in n-SSPP determinations to be somewhat larger, owing to the generally more limited wavelength coverage and (in the present application) lack of available ugriz photometry. An empirical determination of these errors for the n-SSPP is obtained below.

The spectra for our program stars do not reach as far redward as SDSS/SEGUE spectra (hence we cannot use as many of the independent methods as the SSPP provides), and they are of slightly higher resolving power. Thus, during the execution of the n-SSPP, our spectra were first rebinned in order to match the resolving power of SDSS/SEGUE spectra (i.e., to $1 \AA$ linear pixels). The n-SSPP stellar atmospheric parameter estimates are listed in Columns 10-12 of Table 2 as $T_{\text {eff } S}, \log g_{S}$, and $[\mathrm{Fe} / \mathrm{H}]_{S}$, respectively.

The n-SSPP has been modified recently in order to estimate carbon-to-iron abundance ratios (carbonicity, $[\mathrm{C} / \mathrm{Fe}]$ ), based on spectral matching against a dense grid of synthetic spectra. Lee et al. (2013) describe in detail the procedures adopted to estimate $[\mathrm{C} / \mathrm{Fe}]$ for SDSS/SEGUE spectra; these techniques, with different input photometric information, also apply to the n-SSPP. Note that we have recently expanded the carbon grid to reach as low as $[\mathrm{C} / \mathrm{Fe}]=-1.5$, rather than the limit of $[\mathrm{C} / \mathrm{Fe}]=-0.5$ employed by Lee et al. (2013). As shown by Lee et al. (2013), the precision of the carbonicity estimates are better

\footnotetext{
12 If both sets of $V, B-V$, and $J, J-K$ photometry are available, they are used, but the n-SSPP can operate well with one or the other. Even if no photometric measurements are available, the n-SSPP can, in most cases, produce viable stellar parameter estimates (but not distance estimates, which require an input apparent magnitude).
}

Table 3

Carbon Abundance Ratio Estimates

\begin{tabular}{lccccc}
\hline \hline $\begin{array}{l}\text { BM Name } \\
(1)\end{array}$ & $\begin{array}{c}{[\mathrm{C} / \mathrm{Fe}]_{S}} \\
(2)\end{array}$ & $\begin{array}{c}\text { DETECT } \\
(3)\end{array}$ & $\begin{array}{c}\mathrm{CC} \\
(4)\end{array}$ & $\begin{array}{c}{[\mathrm{C} / \mathrm{Fe}]_{H}} \\
(5)\end{array}$ & $\begin{array}{c}{[\mathrm{C} / \mathrm{Fe}]_{C}} \\
(6)\end{array}$ \\
\hline BM-001 & +0.39 & $\mathrm{D}$ & 0.999 & $\ldots$ & +0.19 \\
BM-002 & +0.09 & $\mathrm{D}$ & 0.998 & -0.40 & -0.13 \\
BM-003 & +0.23 & $\mathrm{D}$ & 0.989 & $\ldots$ & +0.03 \\
BM-004 & +0.48 & $\mathrm{D}$ & 0.995 & $\ldots$ & +0.29 \\
BM-005 & +0.61 & $\mathrm{D}$ & 0.994 & +0.26 & +0.43 \\
BM-006 & +0.05 & $\mathrm{D}$ & 1.000 & $\ldots$ & -0.17 \\
BM-007 & +0.14 & $\mathrm{D}$ & 0.998 & -0.15 & -0.07 \\
BM-008 & +0.07 & $\mathrm{D}$ & 0.997 & $\ldots$ & -0.15 \\
BM-009 & +0.28 & $\mathrm{D}$ & 0.999 & $\ldots$ & +0.08 \\
BM-010 & +0.45 & $\mathrm{D}$ & 0.996 & $\ldots$ & +0.26 \\
\hline
\end{tabular}

Notes. CEMP star $\left([\mathrm{C} / \mathrm{Fe}]_{C} \geqslant+0.7\right.$, DETECT $=$ "D," and CC $\left.\geqslant 0.7\right)$.

(This table is available in its entirety in a machine-readable form in the online journal. A portion is shown here for guidance regarding its form and content.)

than 0.35 dex for the parameter space and (generally lower) $\mathrm{S} / \mathrm{N}$ ratios explored by SDSS/SEGUE spectra. We expect similar (or improved) results for application of the n-SSPP to our program spectra, which is checked empirically below. Table 3 lists the medium-resolution estimates of carbonicity, $[\mathrm{C} / \mathrm{Fe}]_{S}$, in Column 2. Column 3 indicates whether the listed measurement is considered a detection, DETECT = "D," lower limit "L," or upper limit "U," and Column 4 provides the correlation coefficient, $\mathrm{CC}$, obtained between the observed spectrum and the best-matching $[\mathrm{C} / \mathrm{Fe}]$ from the model grids. For an acceptable measurement of carbonicity, we demand DETECT = "D" and $\mathrm{CC} \geqslant 0.7$. See Lee et al. (2013) for further discussion of these quantities. There are two stars listed in Table 3 (BM-083 and BM-284) which have CC less than this value; they are marked with a ":" in the DETECT column.

\subsection{Comparison to High-resolution Spectroscopic Analyses}

External measurements of atmospheric parameters and carbon abundances were obtained from various sources in the literature, including the compilations of Cayrel de Strobel et al. (2001), Suda et al. (2008), and Frebel (2010), as well as the references listed in SIMBAD ${ }^{13}$ and in the PASTEL catalog (Soubiran et al. 2010). ${ }^{14}$ In total, we found 170 measurements of $T_{\text {eff }}, 111$ of $\log g, 114$ of [Fe/H], and 50 for [C/Fe]. Note that we have only made use of estimates based on studies published since 1990. A straight average of all available estimates for these parameters was taken (excepting a few instances where it appeared that a given high-resolution estimate was clearly highly discrepant); the results are listed in Columns 13-15 of Table 2 as $T_{\text {eff } H}, \log g_{H}$, and $[\mathrm{Fe} / \mathrm{H}]_{H}$, respectively. Column 4 of Table 3 lists the high-resolution estimates of carbonicity, $[\mathrm{C} / \mathrm{Fe}]_{H}$, for our program stars, where available. Since the range in carbonicity for our program stars with available highresolution determinations is relatively limited, and does not include many stars with $[\mathrm{Fe} / \mathrm{H}]<-2.0$, we have supplemented the comparison sample by obtaining n-SSPP estimates of $[\mathrm{C} / \mathrm{Fe}]_{S}$ from the medium-resolution SDSS/SEGUE spectra for 39 stars with available high-resolution determinations from Aoki et al. (2013).

\footnotetext{
13 http://simbad.u-strasbg.fr/simbad/

14 http://vizier.u-strasbg.fr/viz-bin/VizieR?-source=B/pastel
} 

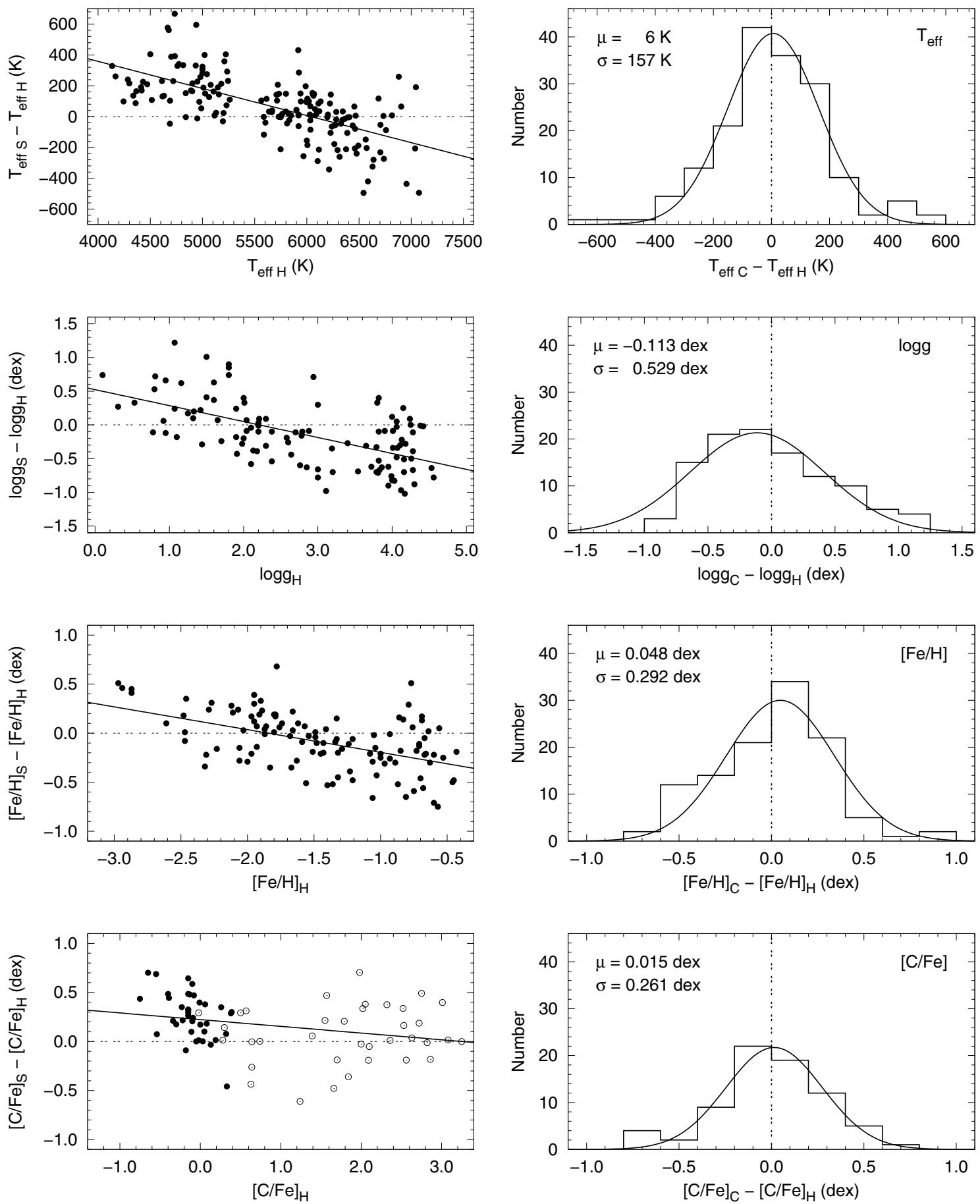

Figure 3. Left panels: differences between the atmospheric parameters and carbon-abundance ratios determined by the $\mathrm{n}-\mathrm{SSPP}, T_{\mathrm{eff}} S, \log g_{S},[\mathrm{Fe} / \mathrm{H}]_{S}$, and $[\mathrm{C} / \mathrm{Fe}]_{S}$, and the values from analyses of high-resolution spectroscopy, $T_{\mathrm{eff} H}, \log g_{H},[\mathrm{Fe} / \mathrm{H}]_{H}$, and $[\mathrm{C} / \mathrm{Fe}]_{H}$ reported in the literature, as a function of the high-resolution spectroscopic values. Filled symbols refer to our B\&M program stars, while in the bottom panel the open symbols represent stars from Aoki et al.(2013), as described in the text. The dashed lines show the linear functions (Equations (1)- (4)) used to obtain corrections to the n-SSPP values, as described in the text. Right panels: histograms of the residuals between the corrected n-SSPP and high-resolution parameters shown in the left panels. Each panel also lists the average offset and scatter determined from a Gaussian fit.

Figure 3 illustrates, in the left-hand column of panels, comparisons of the n-SSPP estimates $T_{\text {eff }}, \log g_{S},[\mathrm{Fe} / \mathrm{H}]_{S}$, and $[\mathrm{C} / \mathrm{Fe}]_{S}$ with the averaged high-resolution spectroscopic results, $T_{\text {eff } H}, \log g_{H},[\mathrm{Fe} / \mathrm{H}]_{H}$, and $[\mathrm{C} / \mathrm{Fe}]_{H}$. The solid lines in these panels are linear fits to the residuals in the difference between the medium- and high-resolution results, as a function of the high-resolution determinations. We use these fits to correct our n-SSPP estimates from the medium-resolution spectra to come into better agreement with the external high-resolution estimates, in the form:

$$
\begin{gathered}
{[\mathrm{Fe} / \mathrm{H}]_{C}=[\mathrm{Fe} / \mathrm{H}]_{S}-\left(-0.232 \times[\mathrm{Fe} / \mathrm{H}]_{S}-0.428\right)} \\
T_{\text {eff } C}=T_{\text {eff } S}-\left(-0.1758 \times T_{\text {eff } S}+1062\right) \\
\log g_{C}=\log g_{S}-\left(-0.237 \times \log g_{S}+0.523\right) \\
{[\mathrm{C} / \mathrm{Fe}]_{C}=[\mathrm{C} / \mathrm{Fe}]_{S}-\left(-0.068 \times[\mathrm{C} / \mathrm{Fe}]_{S}+0.273\right) .}
\end{gathered}
$$



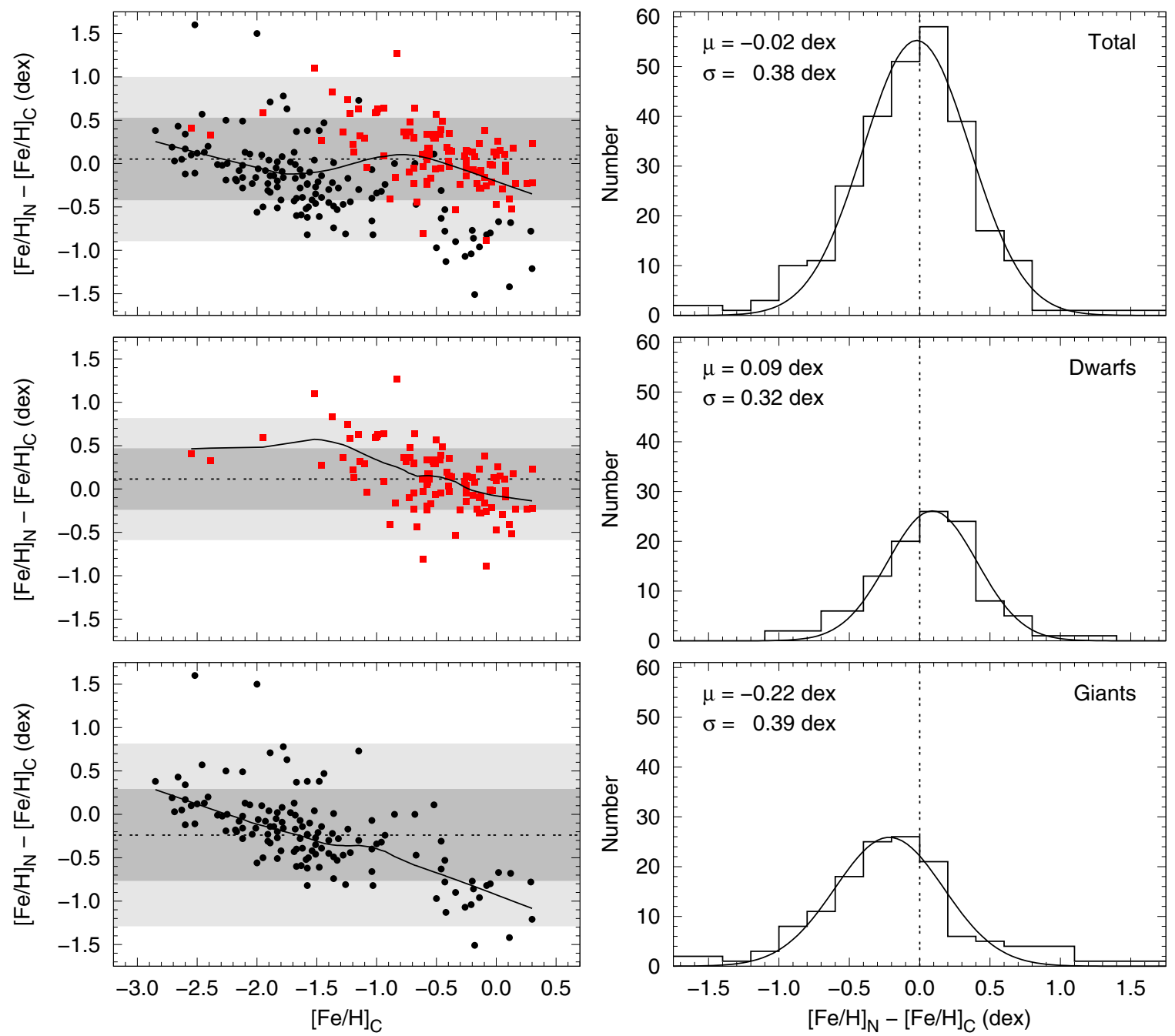

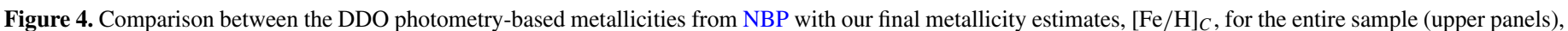

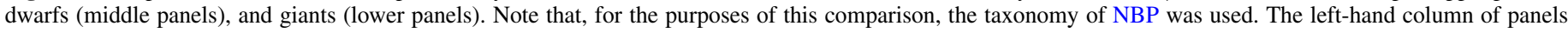

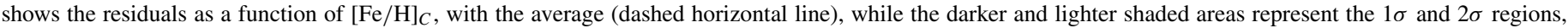

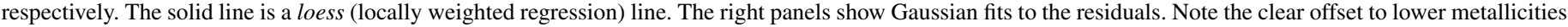
reported by NBP for the giants with metallicities $[\mathrm{Fe} / \mathrm{H}]_{C}>-1.5$ (see the text).

(A color version of this figure is available in the online journal.)

The right-hand column of panels in Figure 3 shows the distribution of residuals between the corrected n-SSPP estimates $\left(T_{\text {eff } C}, \log g_{C}\right.$, and $[\mathrm{Fe} / \mathrm{H}]_{C}$, listed in Columns 16-18 of Table 2 , and $[\mathrm{C} / \mathrm{Fe}]_{C}$, listed in Column 6 of Table 3) and their corresponding high-resolution values. Note that, with the exception of a few individual stars, the agreement is quite satisfactory. Maximum-likelihood Gaussian fits to the distributions of residuals between these various estimates are shown in the right-hand column of panels. Taking into account the expected errors in the (non-uniformly analyzed) highresolution literature estimates of the effective temperature and surface gravity ( $100 \mathrm{~K}$ and $0.35 \mathrm{dex}$, respectively), we conclude that the external accuracies of $T_{\text {eff } C}$ and $\log g_{C}$ are on the order of $125 \mathrm{~K}$ and $0.4 \mathrm{dex}$, respectively. The zero-point offsets of the n-SSPP estimates are acceptably small, on the order of $6 \mathrm{~K}$ and 0.1 dex for $T_{\text {eff } C}$ and $\log g_{C}$, respectively. The rather large external error in the surface gravity estimate is perhaps not surprising, since the spectra do not extend sufficiently redward to include the particularly gravity sensitive $\mathrm{Mg}$ I lines at $\sim 5180 \AA$. Assuming that the expected errors in the literature estimates of metallicity and carbonicity are on the order of $0.20 \mathrm{dex}$ (which may be generous), the external errors in $[\mathrm{Fe} / \mathrm{H}]_{C}$ and $[\mathrm{C} / \mathrm{Fe}]_{C}$ are both $\sim 0.20$ dex.

\subsection{Comparison to the NBP DDO-based Estimates}

We now examine a comparison of our presently determined spectroscopic metallicity estimates with the DDO photometrybased estimates of metallicity given by NBP, and listed as $[\mathrm{Fe} / \mathrm{H}]_{N}$ in Column 19 of Table 2. The upper panels of Figure 4 show the complete sample, while the middle and lower panels for stars classified as dwarfs and giants by NBP, respectively.

The luminosity classes for our program stars are listed in Column 20, in the form $\mathrm{TYPE}_{N / S}$, where " $N$ " indicates those assigned by NBP, while " $S$ " indicates the classes assigned by the n-SSPP. The stars classified as dwarf, subgiant, and red giant map directly onto the classes considered by Beers et al. (2000) (note that we do not discriminate between subgiants and giants in the n-SSPP; they are all classified as giants). We consider the stars NBP classified as blue dwarfs to be main-sequence turnoff (TO) stars, while the blue giant and red horizontal-branch classes 
are considered as field horizontal-branch stars. The UV bright stars are considered to be giants. Note that the classifications are commensurate, in most cases, although they differ for 43 stars (labeled with a “:” in Column 20). The great majority of these conflicting classifications (33 of 43) occur for stars with $T_{\text {eff } C} \geqslant 6000 \mathrm{~K}$, the region where it becomes difficult to distinguish dwarfs from giants close to the main-sequence TO. We proceed with our analysis under the assumption the n-SSPP classification is superior, and make use of it for cases where the luminosity class is in doubt (except where noted).

The average offset and scatter in the metallicity residuals, shown in Figure 4 for the complete sample, are $(\mu, \sigma)=$ $(-0.02$ dex, $0.38 \mathrm{dex})$, while the values for the dwarfs and giants (as classified by NBP) are $(+0.09 \mathrm{dex}, 0.32 \mathrm{dex})$ and $(-0.22 \mathrm{dex}, 0.39 \mathrm{dex})$, respectively. The solid lines in the lefthand panels indicate locally weighted regression (loess) lines that trace the data. As can be appreciated by inspection of the middle panels of Figure 4, the dwarfs exhibit general agreement with our spectroscopic metallicity estimates, with a tendency for $[\mathrm{Fe} / \mathrm{H}]_{N}$ to be somewhat higher than $[\mathrm{Fe} / \mathrm{H}]_{C}$ for metallicities $[\mathrm{Fe} / \mathrm{H}]_{C}<-1.0$. By way of contrast, the $[\mathrm{Fe} / \mathrm{H}]_{N}$ for giants in the lower panels are clearly too low, compared to $[\mathrm{Fe} / \mathrm{H}]_{C}$, for $[\mathrm{Fe} / \mathrm{H}]_{C}>-1.5$, and somewhat higher than $[\mathrm{Fe} / \mathrm{H}]_{C}$ for $[\mathrm{Fe} / \mathrm{H}]_{C}<-1.5$. It is the higher metallicity stars that disagree in the same sense described by Anthony-Twarog \& Twarog (1994) and Ryan \& Lambert (1995), and which were the source of concern for the validity of the original claim for a MWTD. What remains to be shown is whether the existence of a MWTD is supported, or refuted, by the analysis we carry out below, using our improved estimates of metallicity and refined kinematics.

For the remainder of the paper, we drop the subscripts on $[\mathrm{Fe} / \mathrm{H}]_{C}$ and $[\mathrm{C} / \mathrm{Fe}]_{C}$, and simply refer to our adopted metallicity and carbonicity estimates as $[\mathrm{Fe} / \mathrm{H}]$ and $[\mathrm{C} / \mathrm{Fe}]$, respectively.

\section{DISTANCE ESTIMATES AND PROPER MOTIONS}

\subsection{Distance Estimates}

Distances to individual stars in this sample are estimated using the $M_{V}$ versus $(B-V)_{0}$ relationships described by Beers et al. (2000). These relationships require that the likely evolutionary state (luminosity class) of a star be specified. For this, we make use of the taxonomy assigned by the n-SSPP (which assigns types according to the observed surface gravity estimate), the most recent discussion of which is provided by Beers et al. (2012). Note that this includes the reassignment, as necessary, of stars classified as main-sequence TO stars into dwarfs or giants when they otherwise appear in physically impossible positions in the color-magnitude diagram. See Beers et al. (2012) for additional details.

Once types are assigned, distance estimates can be obtained in a straightforward manner. The estimates have to be iterated, because both $V_{0}$ (and therefore the distance estimate) and $(B-V)_{0}$ depend on the adopted reddening. Although the $M_{V}$ versus $(B-V)_{0}$ relationships depend on the metallicity as well, the change in metallicity with small alterations in reddening has little effect. With only a few iterations we obtain consistent estimates of the final reddening, $E(B-V)_{F}$ (listed in Column 11 of Table 1), and the photometric distance estimate, $D_{\text {pho }}$, listed in the fourth column of Table 4 . Based on previous tests of this approach (e.g., Beers et al. 2000, 2012), we expect the photometric distances to be precise to on the order of $10 \%-20 \%$. For most instances, we apply a distance uncertainty of $15 \%$, although in a few cases, larger uncertainties were adopted in order to reflect uncertainties in the determination of reddening corrections.

All but four stars among our program objects have parallaxes available from the Hipparcos astrometric catalog (ESA 1997; van Leeuwen 2007). These parallaxes, and their associated errors, are listed in Columns 2 and 3 of Table 4.

To assess the reliability of our photometric distance estimates, Figure 5 shows the comparison between distances based on photometry, $D_{\text {pho }}$, with the distances based on Hipparcos parallaxes, $D_{\mathrm{HIP}}=1 / \pi_{\mathrm{HIP}}$, for the four different luminosity classes of the targets. Note that this plot only includes stars for which accurate trigonometric and photometric distance estimates are available (that is, we exclude stars with trigonometric parallaxes having $\sigma_{\pi_{\mathrm{HIP}}} / \pi_{\mathrm{HIP}}>0.20$, listed in Column 6 of Table 4, or located at the lowest latitudes, $|b| \leqslant 10^{\circ}$, where reddening, and hence extinction, to a given star is highly uncertain. As can be appreciated by inspection of Figure 5, the relationship between our derived photometric and Hipparcos distances is close to the one-to-one line. Only the few stars with distances greater than about 200 pc exhibit significant scatter.

For the purpose of our kinematic analysis below, we adopt distance estimates based on trigonometric parallaxes, where we judge them to be sufficiently accurate (parallaxes satisfying $\sigma_{\pi_{\mathrm{HIP}}} / \pi_{\mathrm{HIP}} \leqslant 0.20$, and greater than zero). Otherwise, we adopt the derived photometric distance estimate. The final adopted distances, $D_{\text {ado }}$, are listed in Column 7 of Table 4.

\subsection{Proper Motions}

Proper motions for all of our program stars are available from the Hipparcos (ESA 1997; van Leeuwen 2007), Tycho-1 (ESA 1997; Hog et al. 1998), or Tycho-2 (ESA 1997; Høg et al. 2000) catalogs, with average precisions of 1.25 mas $\mathrm{yr}^{-1}$ in $\mu_{\alpha}$ (taking the $\cos \delta$ term into account) and 1.03 mas $\mathrm{yr}^{-1}$ in $\mu_{\delta}$. The precision of the presently available proper motions represent substantial improvements over those used by NBP. Furthermore, while all of our program stars have proper motion estimates, only about one-third of the stars in the NBP catalog had this information available. Columns 8 and 9 of Table 4 list the proper motions for our program stars, while Columns 10 and 11 present their associated errors. The final column lists the identifier of the star in the Hipparcos or Tycho catalogs.

\section{KINEMATIC ANALYSIS OF THE B\&M SAMPLE}

In this section we examine the kinematic properties of the B\&M sample of stars studied by NBP.

Figure 6 shows the distribution of the absorption-corrected $V_{0}$ magnitudes, dereddened $(B-V)_{0}$ colors, adopted distances, $D_{\text {ado }}$, and estimates of metallicities, $[\mathrm{Fe} / \mathrm{H}]$, for our sample of program stars. As is immediately clear from inspection of this figure, this is a very local sample of stars, with $\sim 90 \%$ of the stars located within $1 \mathrm{kpc}$ from the Sun. Nevertheless, because of the metallicity bias in the original selection, some $70 \%$ have $[\mathrm{Fe} / \mathrm{H}] \leqslant-0.5$, suitable for exploration of the thick disk, MWTD, and inner-halo population. There are not large numbers of stars in this sample with $[\mathrm{Fe} / \mathrm{H}]<-2.0(\sim 10 \%$ of the sample), which limits its utility for examination of the outerhalo population.

\subsection{Determination of Space Motions and Orbital Parameters}

It is our intention to obtain the most precise kinematics for our program stars, taking advantage of the refinements that more 


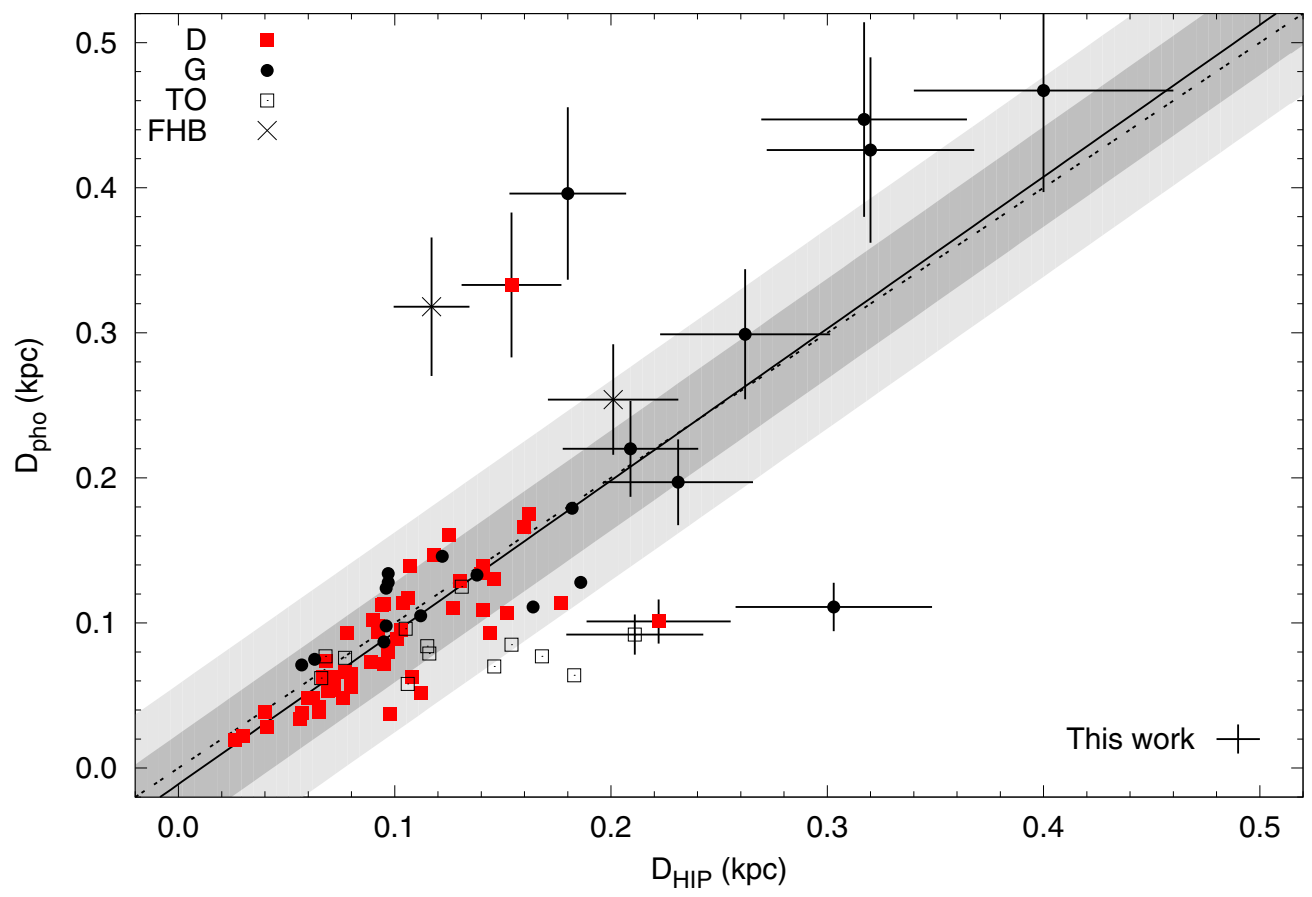

Figure 5. Comparison of the photometrically estimated distances, $D_{\text {pho }}$, with the trigonometric distance estimates, $D_{\text {HIP }}$, for stars with sufficiently accurate Hipparcos parallaxes $\left(\sigma_{\pi_{\mathrm{HIP}}} / \pi_{\mathrm{HIP}} \leqslant 0.20\right)$. The dashed line is the one-to-one line, while the solid line is a robust regression fit to the data. The darker and lighter shaded areas represent the $1 \sigma$ and $2 \sigma$ regions about the linear fit, respectively, based on a Gaussian fit to the residuals. The error bar in the lower right corner of the plot is the typical error for stars with distances less than $200 \mathrm{pc}$. For stars with distances (either photometric or astrometric) greater than $200 \mathrm{pc}$, individual error bars are shown. (A color version of this figure is available in the online journal.)

Table 4

Parallaxes, Distance Estimates, and Proper Motions

\begin{tabular}{|c|c|c|c|c|c|c|c|c|c|c|c|c|}
\hline $\begin{array}{l}\text { BM Name } \\
\text { (1) }\end{array}$ & $\begin{array}{c}\pi_{\mathrm{HIP}} \\
(\mathrm{mas}) \\
(2)\end{array}$ & $\begin{array}{c}\sigma_{\pi_{\mathrm{HIP}}} \\
(\mathrm{mas}) \\
(3)\end{array}$ & $\begin{array}{c}D_{\text {pho }} \\
(\mathrm{kpc}) \\
(4)\end{array}$ & $\begin{array}{c}D_{\mathrm{HIP}} \\
(\mathrm{kpc}) \\
(5)\end{array}$ & $\begin{array}{c}\sigma_{\pi_{\mathrm{HIP}}} / \pi_{\mathrm{HIP}} \\
(6)\end{array}$ & $\begin{array}{c}D_{\text {ado }} \\
(\mathrm{kpc}) \\
(7)\end{array}$ & $\begin{array}{c}\sigma_{\mathrm{D}_{\mathrm{ado}}} \\
(\mathrm{kpc}) \\
(8)\end{array}$ & $\begin{array}{c}\mu_{\alpha} \\
\left(\operatorname{mas}_{y r^{-1}}\right) \\
(9)\end{array}$ & $\begin{array}{c}\mu_{\delta} \\
\left(\operatorname{mas~yr}^{-1}\right) \\
(10)\end{array}$ & $\begin{array}{c}\sigma_{\mu_{\alpha}} \\
\left(\operatorname{mas~yr}^{-1}\right) \\
(11)\end{array}$ & $\begin{array}{c}\sigma_{\mu_{\delta}} \\
\left(\operatorname{mas~yr}^{-1}\right) \\
(12)\end{array}$ & HIP/TYCHO ID \\
\hline BM-001 & 6.71 & 1.40 & 0.097 & 0.149 & 0.21 & 0.097 & 0.015 & 8.82 & 23.43 & 1.50 & 0.80 & 921 \\
\hline BM-002 & 0.88 & 0.81 & 0.530 & 1.136 & 0.92 & 0.530 & 0.080 & -1.25 & -51.79 & 0.90 & 0.59 & 2463 \\
\hline BM-003 & 9.51 & 1.11 & 0.096 & 0.105 & 0.12 & 0.105 & 0.012 & 20.94 & -546.76 & 1.31 & 0.79 & 3026 \\
\hline BM-004 & 4.24 & 1.14 & 0.133 & 0.236 & 0.27 & 0.133 & 0.020 & 61.47 & -22.35 & 1.23 & 0.69 & 3134 \\
\hline BM-005 & 5.01 & 1.32 & 0.547 & 0.200 & 0.26 & 0.547 & 0.082 & 60.61 & 21.00 & 1.35 & 0.94 & 3554 \\
\hline BM-006 & 3.19 & 0.79 & 0.115 & 0.314 & 0.25 & 0.115 & 0.017 & 63.61 & 49.81 & 0.92 & 0.50 & 5104 \\
\hline BM-007 & 7.23 & 0.86 & 0.133 & 0.138 & 0.12 & 0.138 & 0.016 & 134.10 & 12.53 & 0.70 & 0.97 & 5455 \\
\hline BM-008 & 10.56 & 1.01 & 0.072 & 0.095 & 0.10 & 0.095 & 0.009 & 284.85 & -8.13 & 0.93 & 0.72 & 5489 \\
\hline BM-009 & 15.35 & 1.17 & 0.042 & 0.065 & 0.08 & 0.065 & 0.005 & -230.98 & -459.24 & 1.14 & 0.87 & 6159 \\
\hline BM-010 & 7.61 & 1.38 & 0.125 & 0.131 & 0.18 & 0.131 & 0.024 & 55.01 & -16.16 & 1.43 & 0.91 & 6164 \\
\hline
\end{tabular}

(This table is available in its entirety in a machine-readable form in the online journal. A portion is shown here for guidance regarding its form and content.)

modern data have provided in the 28 years since the publication of the first paper in this series.

Adopted distances are determined as described in Subsection 4.1 above. Proper motions, almost all taken from the Hipparcos catalog, are described in Section 4.2 above. From Table 2, we adopt the high-resolution spectroscopic determinations of RVs, $\mathrm{RV}_{H}$, where available, which are expected to have precisions of $2 \mathrm{~km} \mathrm{~s}^{-1}$ or better. When not available, we make use of the determinations based on medium-resolution spectroscopy, which were demonstrated in Section 2.3 to exhibit precisions on the order of $5 \mathrm{~km} \mathrm{~s}^{-1}$.

We now derive the space motions and orbital parameters of our program stars, following the procedures described by Carollo et al. (2010).

Corrections for the motion of the Sun with respect to the local standard of rest (LSR) are applied during the course of the calculation of the full space motions; here we adopt the values $(U, V, W)=(9,12,7) \mathrm{km} \mathrm{s}^{-1}$ (Mihalas \& Binney 1981). Note that we follow the convention that $U$ is positive in the direction away from the Galactic center, $V$ is positive in the direction of Galactic rotation, and $W$ is positive toward the north Galactic pole. For the purpose of this analysis it is also convenient to obtain the rotational component of a star's motion about the Galactic center in a cylindrical frame, denoted as $V_{\phi}$, and calculated assuming that the LSR is on a circular orbit with a value of $220 \mathrm{~km} \mathrm{~s}^{-1}$ (Kerr \& Lynden-Bell 1986). Our assumed values of $R_{\odot}(8.5 \mathrm{kpc})$ and the circular velocity of the LSR are both consistent with two recent independent determinations of these quantities by Ghez et al. (2008) and Koposov et al. (2009). Bovy et al. (2012d) recently obtained an estimate of the Milky Way's circular velocity at the position of the Sun of $V_{c}\left(R_{\odot}\right)=218 \pm 6 \mathrm{~km} \mathrm{~s}^{-1}$, based on an analysis of 

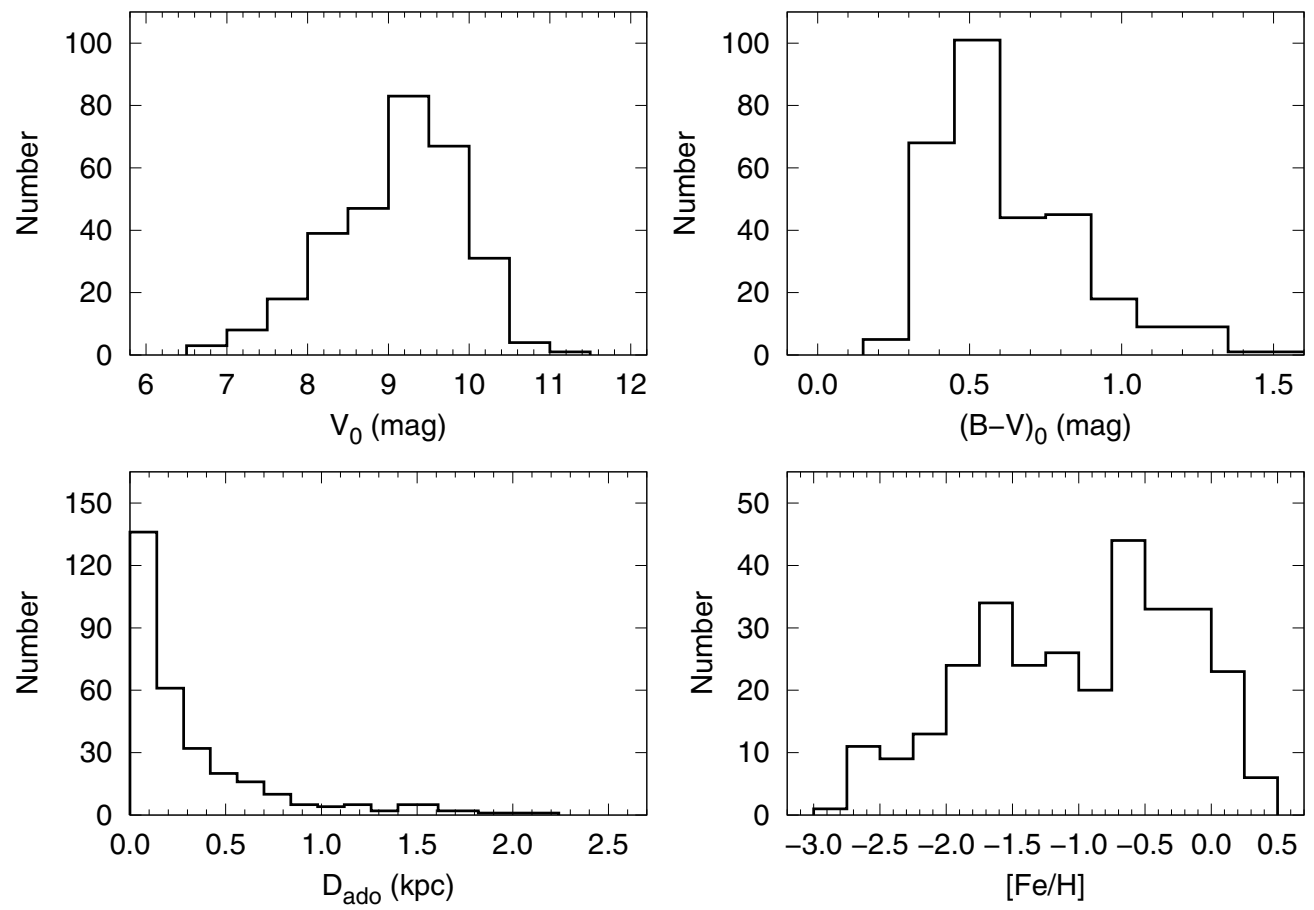

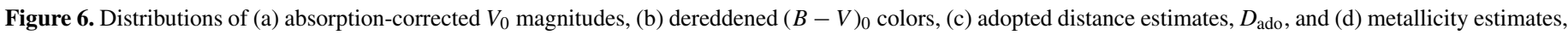
$[\mathrm{Fe} / \mathrm{H}]$, for our program stars.

high-resolution spectroscopic determinations from the Apache Point Observatory Galactic Evolution Experiment (APOGEE; Majewski et al. 2010), part of the Sloan Digital Sky Survey III (SDSS-III; Eisenstein et al. 2011), which is also consistent with our adopted value.

The orbital parameters of the stars, such as the perigalactic distance (the closest approach of an orbit to the Galactic center), $r_{\text {peri }}$, the apogalactic distance (the farthest extent of an orbit from the Galactic center), $r_{\text {apo }}$, of each stellar orbit, and the orbital eccentricity, $e$, defined as $e=\left(r_{\text {apo }}-r_{\text {peri }}\right) /\left(r_{\text {apo }}+r_{\text {peri }}\right)$, as well as $Z_{\max }$ (the maximum distance that a stellar orbit achieves above or below the Galactic plane), are derived by adopting an analytic Stäckel-type gravitational potential (which consists of a flattened, oblate disk, and a nearly spherical massive darkmatter halo; see the description given by Chiba \& Beers 2000, Appendix A), and integrating their orbital paths based on the starting point obtained from the observations.

Table 5 provides a summary of the above calculations. Column 1 provides the star names. Columns 2 and 3 list the positions of the stars in the meridional $(R, Z)$-plane. The derived $U V W$ velocity components are provided in Columns 4-6; their associated errors are listed in Columns 7-9. Column 10 lists the velocity projected onto the Galactic plane $\left(V_{R}\right.$, positive in the direction away from the Galactic center), while Column 11 lists the derived rotation velocity, $V_{\phi}$. The derived $r_{\text {peri }}$ and $r_{\text {apo }}$ are given in Columns 12 and 13, respectively. Columns 14 and 15 list the derived $Z_{\max }$ and orbital eccentricity, $e$, respectively.

Errors on our derived estimates of the individual components of the space motions take into account an estimated $15 \%$ error in the photometric distances (individual errors in Hipparcos distances, when adopted, are used), as well as the individual errors in the proper motions and the adopted RVs $\left(2 \mathrm{~km} \mathrm{~s}^{-1}\right.$ for the high-resolution determinations, $5 \mathrm{~km} \mathrm{~s}^{-1}$ for the mediumresolution determinations). As expected, when compared to the previous results of NBP, the derived errors in these quantities are much improved. Figure 7 shows the distributions of these errors for both sets of analyses. After removing the stars with individual estimated errors in any one of the three components of space motion larger than $50 \mathrm{~km} \mathrm{~s}^{-1}$ from each of these samples (or which were dropped for other reasons), the average errors for the B\&M sample are $\sigma(U, V, W)=(7.9,9.1,6.5) \mathrm{km} \mathrm{s}^{-1}$. For NBP, the average errors were $2-2.5$ times as high, $\sigma(U, V, W)=$ $(19.3,19.7,16.8) \mathrm{km} \mathrm{s}^{-1}$. The large errors in the individual space motions (and eccentricities) of some stars forced NBP to rather severely trim their sample from which inferences could be made about the nature of the underlying populations.

A total of 42 stars in our full sample of 302 stars are not used in the kinematic analysis, because they are either missing one or more of the input quantities used for the determination of their space motions, are located at Galactic latitudes $|b|<$ $10^{\circ}$ and had only photometric distances available (and hence uncertain estimates of reddening), or had individual estimated errors in any one of the three components of space motion larger than $50 \mathrm{~km} \mathrm{~s}^{-1}$. Such stars are noted in the final column of Table 5, where the first digit of the INOUT parameter set to " 0 " indicates that the star has been dropped from subsequent kinematical analysis.

\subsection{Distributions of $U V W$, and $Z_{\max }$ versus $[\mathrm{Fe} / \mathrm{H}]$}

Figure 8 presents the individual components of the space motions, as a function of $[\mathrm{Fe} / \mathrm{H}]$, for our program stars with accepted kinematic estimates. It is clear from inspection of this diagram that there exists a "core" of stars with relatively high net rotation and low velocity dispersion down to at least $[\mathrm{Fe} / \mathrm{H}]=$ -1.3 , and possibly a little lower. This immediately suggests the presence of low-metallicity stars in the disk system, well below the mean abundance typically associated with the canonical thick disk, on the order of $[\mathrm{Fe} / \mathrm{H}]=-0.6$.

Since the great majority of our program stars are located within $1 \mathrm{kpc}$, it is difficult to separate possible stellar populations on the basis of vertical distance from the Galactic plane. 

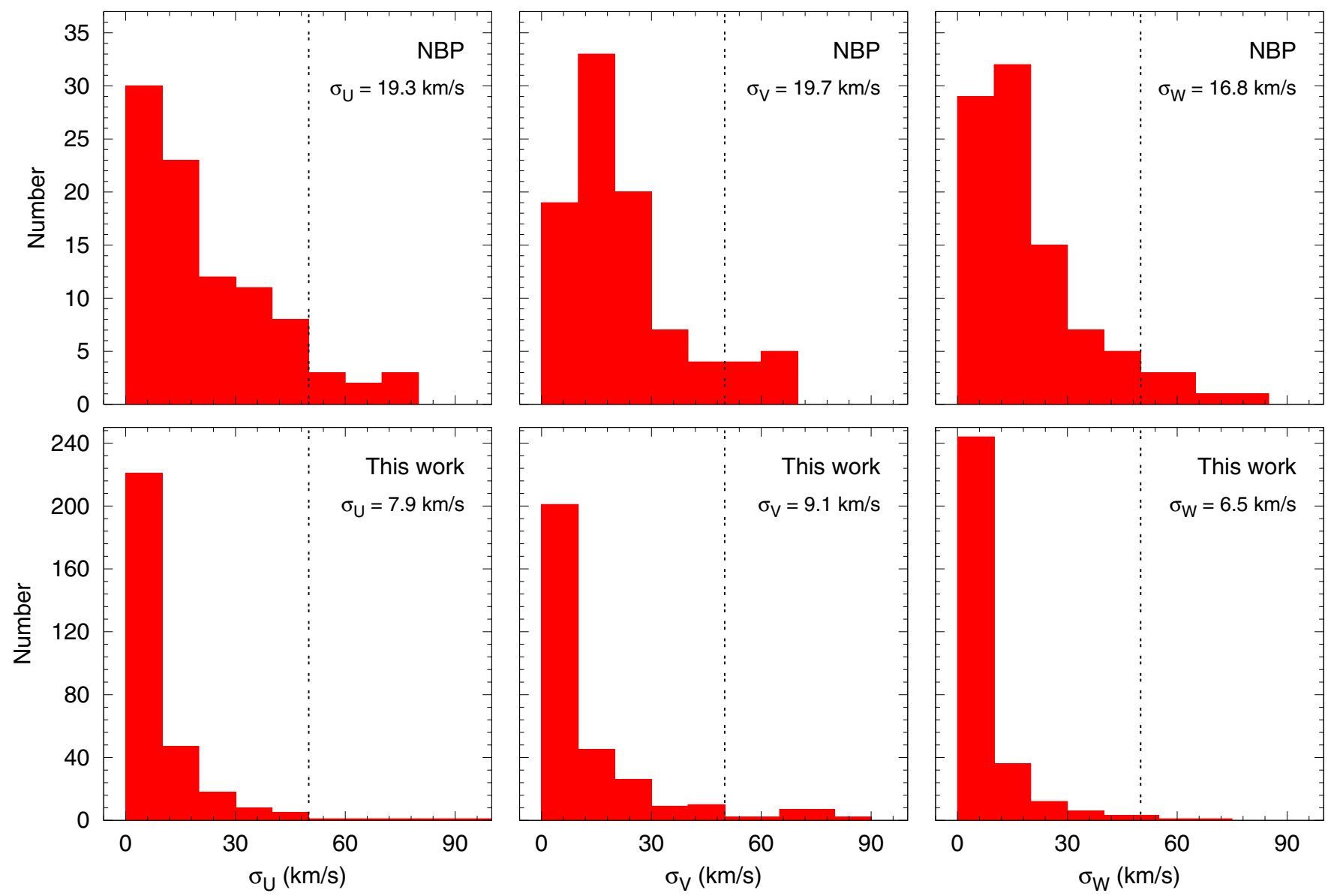

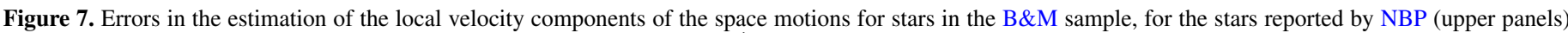

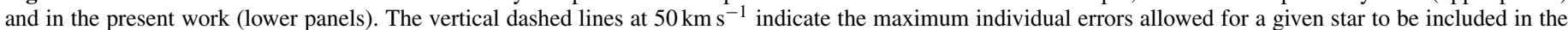

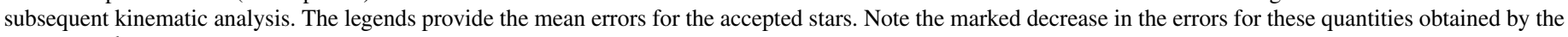
present work.

(A color version of this figure is available in the online journal.)

Table 5

Space Motions and Orbital Parameters

\begin{tabular}{|c|c|c|c|c|c|c|c|c|c|c|c|c|c|c|c|}
\hline BM Name & $\begin{array}{c}R \\
(\mathrm{kpc}) \\
(2)\end{array}$ & $\begin{array}{c}Z \\
(\mathrm{kpc}) \\
(3)\end{array}$ & $\begin{array}{c}U \\
\left(\mathrm{~km} \mathrm{~s}^{-1}\right) \\
(4)\end{array}$ & $\begin{array}{c}V \\
\left(\mathrm{~km} \mathrm{~s}^{-1}\right) \\
(5)\end{array}$ & $\begin{array}{c}W \\
\left(\mathrm{~km} \mathrm{~s}^{-1}\right) \\
(6)\end{array}$ & $\begin{array}{c}\sigma(U) \\
\left(\mathrm{km} \mathrm{s}^{-1}\right) \\
(7)\end{array}$ & $\begin{array}{c}\sigma(V) \\
\left(\mathrm{km} \mathrm{s}^{-1}\right) \\
(8)\end{array}$ & $\begin{array}{c}\sigma(W) \\
\left(\mathrm{km} \mathrm{s}^{-1}\right) \\
(9)\end{array}$ & $\begin{array}{c}V_{\mathrm{R}} \\
\left(\mathrm{km} \mathrm{s}^{-1}\right) \\
(10)\end{array}$ & $\begin{array}{c}V_{\phi} \\
\left(\mathrm{km} \mathrm{s}^{-1}\right) \\
(11)\end{array}$ & $\begin{array}{c}r_{\text {peri }} \\
(\mathrm{kpc}) \\
(12)\end{array}$ & $\begin{array}{c}r_{\text {apo }} \\
(\mathrm{kpc}) \\
(13)\end{array}$ & $\begin{array}{c}Z_{\max } \\
(\mathrm{kpc}) \\
(14)\end{array}$ & $\begin{array}{c}e \\
(15)\end{array}$ & $\overline{\text { INOUT }}$ \\
\hline BM-001 & 8.503 & -0.091 & 1 & 34 & -32 & 2 & 2 & 5 & 2 & 254 & 8.50 & 11.41 & 0.55 & 0.15 & $1 / 1 / 1$ \\
\hline BM-002 & 8.514 & -0.520 & -80 & -107 & 43 & 11 & 16 & 4 & -78 & 113 & 3.05 & 9.35 & 1.01 & 0.51 & $1 / 1 / 0$ \\
\hline BM-003 & 8.514 & -0.099 & -144 & -225 & -36 & 15 & 25 & 10 & -144 & -4 & 0.10 & 10.54 & 0.69 & 0.98 & $1 / 0 / 0$ \\
\hline BM-004 & 8.511 & -0.130 & 19 & -13 & -26 & 4 & 5 & 5 & 19 & 206 & 7.30 & 8.74 & 0.37 & 0.09 & $1 / 1 / 0$ \\
\hline BM-005 & 8.579 & -0.521 & 143 & -45 & 82 & 24 & 7 & 2 & 145 & 171 & 4.88 & 13.03 & 3.33 & 0.46 & $1 / 0 / 0$ \\
\hline BM-006 & 8.521 & -0.111 & 36 & 15 & 7 & 6 & 1 & 1 & 36 & 235 & 7.98 & 10.29 & 0.17 & 0.13 & $1 / 1 / 2$ \\
\hline BM-007 & 8.469 & -0.120 & 40 & -84 & -93 & 9 & 5 & 1 & 38 & 136 & 4.44 & 8.81 & 2.40 & 0.33 & $1 / 1 / 0$ \\
\hline BM-008 & 8.521 & -0.091 & 104 & -55 & -29 & 10 & 7 & 5 & 104 & 164 & 4.53 & 10.45 & 0.46 & 0.40 & $1 / 1 / 0$ \\
\hline BM-009 & 8.517 & -0.061 & -143 & -58 & -40 & 10 & 5 & 4 & -142 & 161 & 4.16 & 11.96 & 0.82 & 0.48 & $1 / 0 / 0$ \\
\hline BM-010 & 8.528 & -0.127 & 18 & -13 & -13 & 4 & 5 & 1 & 18 & 206 & 7.30 & 8.72 & 0.21 & 0.09 & $1 / 1 / 1$ \\
\hline
\end{tabular}

Notes. INOUT indicates membership in various subsamples considered in this paper (see the text). The first digit takes on a value of " 1 " if the star is accepted for the kinematic analysis, " 0 " if not. The second digit takes on a value of " 1 " if the star is likely to be a member of the disk system, according to the Lindblad diagram shown in Figure 12, " 0 " if not. The third digit takes on a value of " 1 " if the star is a likely disk member with metallicity in the range $-0.8<[\mathrm{Fe} / \mathrm{H}] \leqslant-0.5$, " 2 " if it is a likely disk member in the metallicity range $-1.8<[\mathrm{Fe} / \mathrm{H}] \leqslant-0.8$, " 0 " if not.

(This table is available in its entirety in a machine-readable form in the online journal. A portion is shown here for guidance regarding its form and content.)

As an alternative, we have explored using the derived maximum distance from the plane, $Z_{\max }$. Figure 9 shows the result of this exercise, where we have plotted the base- $10 \log$ of $Z_{\max }$ as a function of $[\mathrm{Fe} / \mathrm{H}]$. A reference line at $Z_{\max }=3 \mathrm{kpc}$ is shown.
From inspection of this figure, it is clear that significant numbers of stars with $Z_{\max }>3 \mathrm{kpc}$ are not found for $[\mathrm{Fe} / \mathrm{H}] \gtrsim-1.5$. Although some overlap between the inner-halo population and the proposed MWTD population is unavoidable, we expect the 

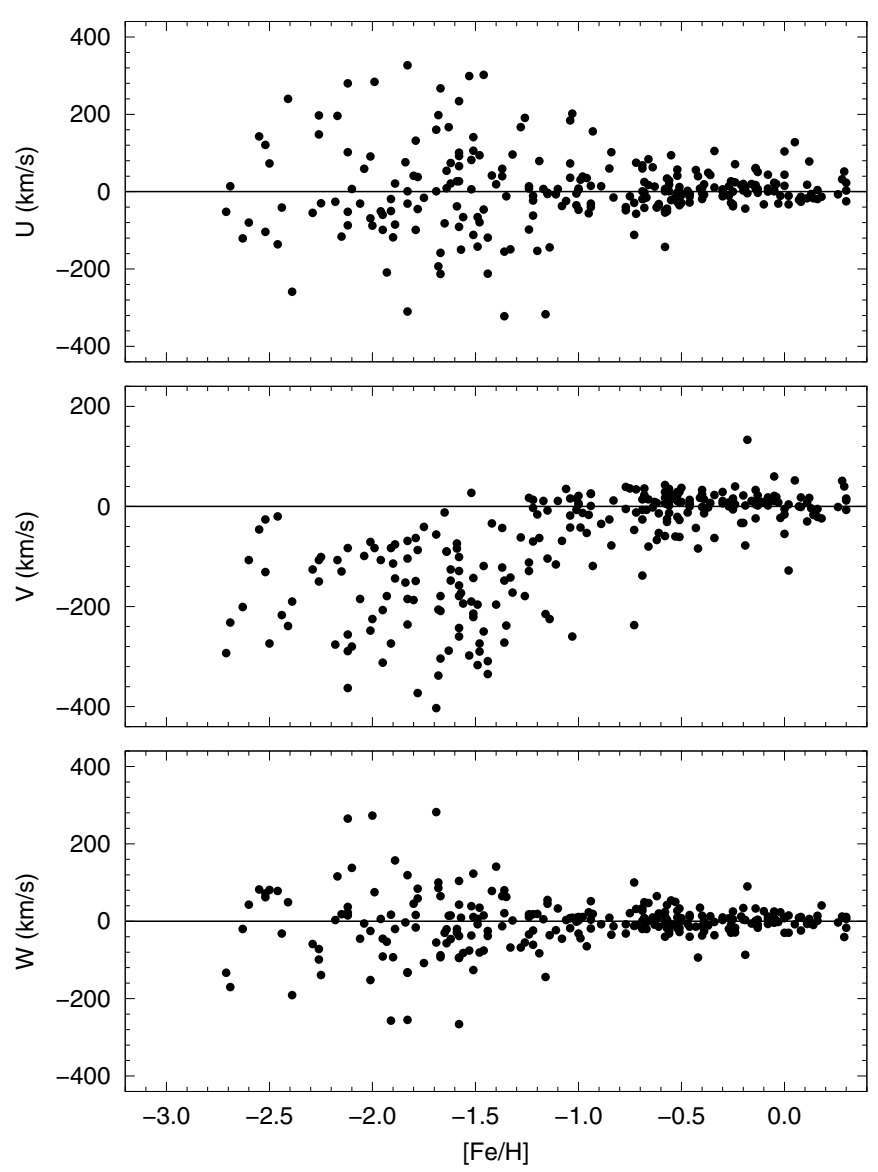

Figure 8. Local velocity components for stars in the B\&M sample with available $U V W$ estimates, as a function of metallicity, $[\mathrm{Fe} / \mathrm{H}]$. Note the existence of stars with low-velocity dispersions in their estimated components down to at least $[\mathrm{Fe} / \mathrm{H}=-1.5]$, and possibly a little lower. Stars with errors in any of the individual derived components of motion exceeding $50 \mathrm{~km} \mathrm{~s}^{-1}$ are excluded.

majority of the stars with $Z_{\max } \leqslant 3 \mathrm{kpc}$ and in the metallicity interval $-1.8 \leqslant[\mathrm{Fe} / \mathrm{H}] \leqslant-0.8$ (following Carollo et al. 2010) to be associated with the MWTD. As is shown below, stars in this metallicity interval also exhibit a lower net rotation than the stars of the canonical thick disk.

\subsection{The $[\mathrm{Fe} / \mathrm{H}]$ versus Eccentricity Diagram}

One of the central arguments of NBP, that there exist numerous stars in the B\&M sample (where none were found in the sample used by ELS to support their classic monolithic collapse model) in the regime $[\mathrm{Fe} / \mathrm{H}] \leqslant-1.0, e \leqslant 0.4$, can now be re-examined using our improved kinematic results. The upper panel of Figure 10 is a plot of $[\mathrm{Fe} / \mathrm{H}]$, as a function of orbital eccentricity, for the sample of B\&M stars from NBP (their Figure 14), where we have used their derived metallicities and eccentricities (restricted, as did they, to stars with estimated errors in the eccentricity $\leqslant 0.1)$. The lower panel of the figure shows the more comprehensive results for the B\&M stars from the present analysis. All of our program stars with acceptable kinematic determinations have estimated errors in eccentricity less than 0.1. In both panels, for heuristic purposes, the red squares stand for red giants (NBP, upper panel) and giants (this work, lower panels), while black circles are used for all other objects. The two panels also show the original box $(-2.0 \leqslant$ $[\mathrm{Fe} / \mathrm{H}] \leqslant-1.0,0.2 \leqslant e \leqslant 0.4)$ used by NBP to contrast the locations of the stars in the ELS sample with their own. Note that there are roughly 10 stars in the NBP analysis that fall in the box (plus or minus a few that are right at the edges of the box) compared with none in the original ELS sample. As seen in the lower panel, the present analysis now includes almost twice as many stars in the same box.

Clearly, the addition of more stars with improved estimates of metallicities and eccentricities in the present sample strengthens the original thesis of NBP that the ELS model was incompatible with results obtained for the B\&M sample of non-kinematically selected stars.

Of course, more modern analyses of even larger such samples (e.g., Chiba \& Beers 2000; Carollo et al. 2007, 2010) have come to similar conclusions, and are consistent with the expectations from contemporary hierarchical galaxy-assembly models. However, this idea had its observational origin for field stars in the work of $\mathrm{NBP}^{15}$.

\subsection{The Toomre and Lindblad Diagrams}

The so-called Toomre diagram (a plot of $\left(U^{2}+W^{2}\right)^{1 / 2}$, the quadratic addition of the $U$ and $W$ velocity components, as a function of the rotational component, $V$ ) and the Lindblad diagram (a plot of the integrals of motion representing the total energy, $E$, and the vertical angular momentum component, $L_{Z}$ ) are commonly used to investigate the nature of the kinematics of stellar populations in the Galaxy. Given the high quality of the estimated kinematics for the B\&M sample, it is useful to examine these diagrams to glean any insight we can from them.

For the sake of comparison, we have also derived these diagrams based on the sample of RAVE stars reported by Ruchti et al. (2011). ${ }^{16}$ The Ruchti et al. sample is of similar size, and covers a similar metallicity range as the B\&M sample, but carries along its own set of biases in the selection of the member stars (see Ruchti et al. 2010, for a discussion). In addition, the proper motions available to Ruchti et al. are not as precise, in general, as those in our sample, since few of their stars were included in the Hipparcos, Tycho, or Tycho-2 catalogs.

Figure 11 shows the Toomre diagrams for the two samples. The upper panel is the sample of Ruchti et al. (after removal of the 72 of 319 stars that are either missing the input quantities used for determination of their space motions, or that have individual estimated errors in any one of the three components of space motion larger than $50 \mathrm{~km} \mathrm{~s}^{-1}$ ). Following the removal of these stars, the average errors in the derived space motions are $\sigma(U, V, W)=(14.4,15.6,12.2) \mathrm{km} \mathrm{s}^{-1}$, about twice as high as from our analysis of the B\&M sample, but still better than obtained from the NBP analysis.

The lower panel shows the Toomre diagram for the B\&M sample. The legend indicates the metallicity intervals that are distinguished in the two panels. These were chosen to roughly separate stars expected to belong to the thick (or thin) disk $([\mathrm{Fe} / \mathrm{H}]>-0.8)$, the suggested MWTD $(-1.8<$ $[\mathrm{Fe} / \mathrm{H}] \leqslant-0.8)$, and the halo system $([\mathrm{Fe} / \mathrm{H}] \leqslant-1.8)$, taking our guidance in selecting these intervals from Carollo et al. (2010). As expected, the more metal-rich stars in both samples

\footnotetext{
15 J.E.N. acknowledges here the validity of the criticism of the NBP abundances for red giants by Anthony-Twarog \& Twarog (1994) and Ryan \& Lambert (1995); for historians of science, he notes that the discrepancy results from errors in the limited DDO abundance calibration adopted for (absolutely) fainter, more metal-rich giants. That said, inspection of the present Figure 10 shows that, with the present abundance calibration, giants exist in the region where ELS (due to their selection criteria) found no stars.

16 We have updated the estimates of $[\mathrm{Fe} / \mathrm{H}]$ and the derived $U V W$ used for the Ruchti et al. sample based on revised information kindly supplied by G. Ruchti (2013, private communication), based on a newer version of the RAVE pipeline outputs.
} 


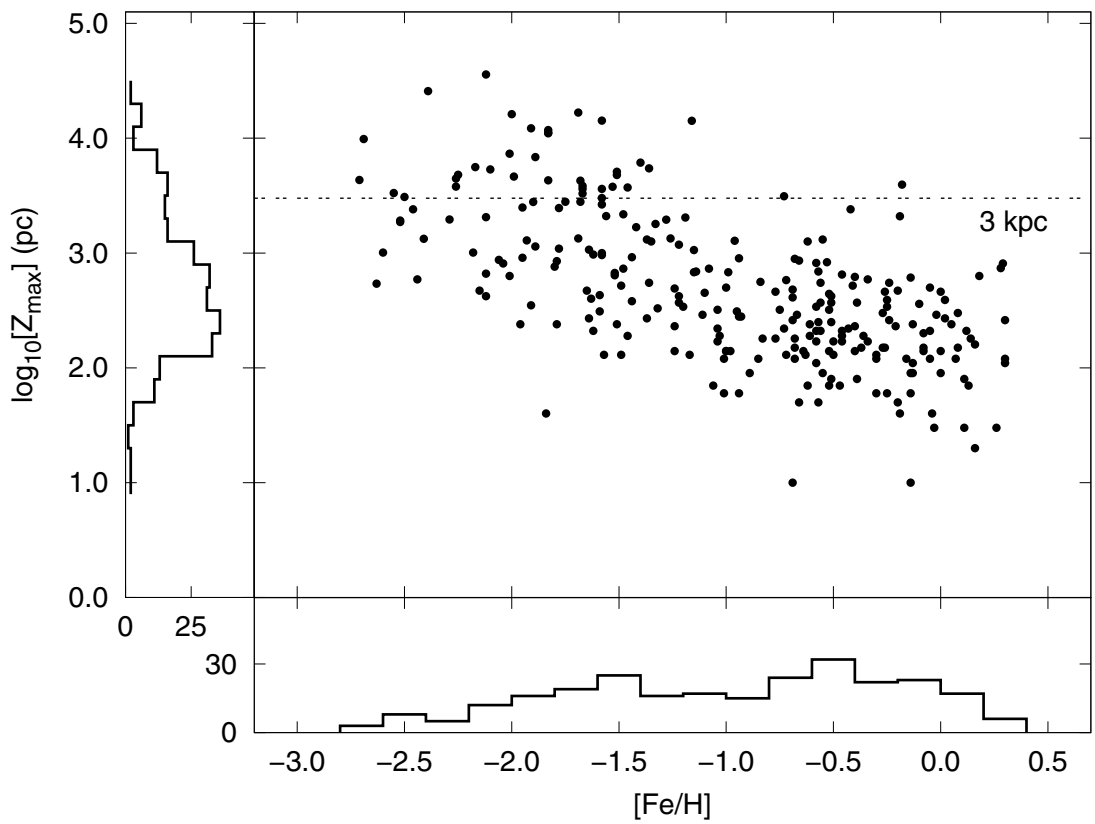

Figure 9. Distribution of $Z_{\max }$, the largest distance above or below the Galactic plane achieved by a star during the course of its orbit, as a function of metallicity, for the stars in the B\&M sample. The marginal distributions of each variable are shown as histograms. The horizontal dashed line provides a reference at $3 \mathrm{kpc}$. Very few stars with metallicity $[\mathrm{Fe} / \mathrm{H}]>-1.5$ achieve orbits that reach higher than this location. Note the logarithmic scale for $Z_{\max }$. Stars with errors in any of the individual derived components of motion exceeding $50 \mathrm{~km} \mathrm{~s}^{-1}$ are excluded.
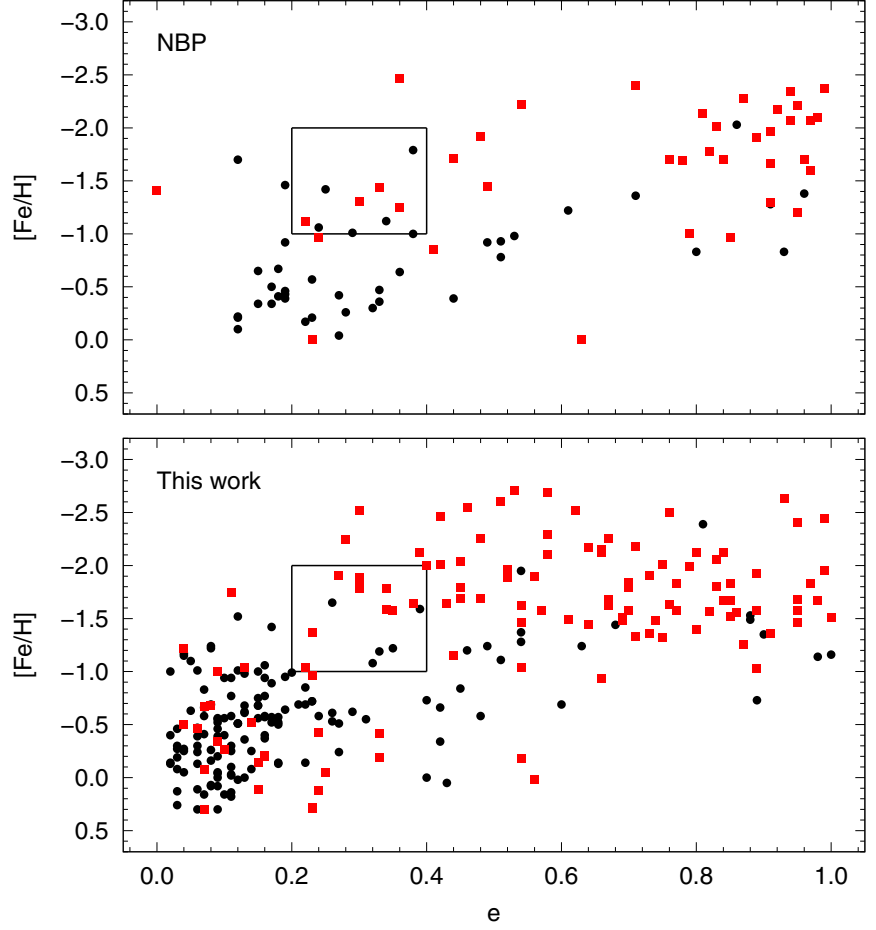

Figure 10. Distribution of metallicity, $[\mathrm{Fe} / \mathrm{H}]$, for stars in the B\&M sample, as a function of derived orbital eccentricity, $e$. In both panels the red squares stand for red giants while black circles are used for all other objects. Upper panel: the sample studied by NBP (and adopting their metallicities and eccentricities), which only included stars with eccentricities having errors less than 0.1 . The box indicates the region of interest originally considered by NBP. Lower panel: the present sample, all of which have errors in eccentricity less than 0.1. In addition to a much larger total sample, note that the box now contains about twice as many stars as for the NBP sample. Stars with errors in any of the individual derived components of motion exceeding $50 \mathrm{~km} \mathrm{~s}^{-1}$ are excluded.

(A color version of this figure is available in the online journal.) are primarily found in the region with low $\left(U^{2}+W^{2}\right)^{1 / 2}$ and high orbital rotation velocities $\left(U^{2}+W^{2}\right)^{1 / 2} \lesssim 100 \mathrm{~km} \mathrm{~s}^{-1}$, $-100<V<100 \mathrm{~km} \mathrm{~s}^{-1}$ ), while stars with intermediate metallicities are divided between those inside and outside this region.

The Lindblad diagrams for these two samples of stars, calculated following the prescription described by Carollo et al. (2014), are shown in Figure 12. As can be appreciated from inspection of the left-hand panel, which applies to the B\&M sample, there is rather clear separation of a region corresponding to a rotationally supported disk system, indicated by the dashed line shown to guide the eye. The same line is drawn in the right-hand panel, which applies to the Ruchti et al. sample. The stars to the right of the line are expected to be dominated by members of the disk system (thin, thick, and MWTD) while those to the left of the line are likely to be dominated by innerhalo members. Note that the separation of stars across this line is somewhat less clear for the Ruchti et al. sample, presumably because of the larger errors in the derived kinematics. Also note that this kinematic division does not isolate only the more metalrich stars, but in both samples appears to include relatively large fractions of stars in the intermediate metallicity interval, $-1.8<$ $[\mathrm{Fe} / \mathrm{H}] \leqslant-0.8$, and a few stars with even lower metallicity.

\subsection{Distributions of $V_{\phi}$ for the B\&M and Ruchti et al. Samples}

We now examine, in greater detail, the motions of the stars isolated above by their location in the Lindblad diagrams. The top row of the upper grouping of panels in Figure 13 shows stripe density plots of the distribution of $V_{\phi}$ for the full sets of stars with metallicities $-0.8<[\mathrm{Fe} / \mathrm{H}] \leqslant-0.5$ from the B\&M (left) and Ruchti et al. (right) samples. The lower row in this grouping shows the stars in this same metallicity interval selected to the right of the segregation line in Figure 12, labeled as the "disk sample." This metallicity interval is expected to primarily comprise thick-disk stars, but 

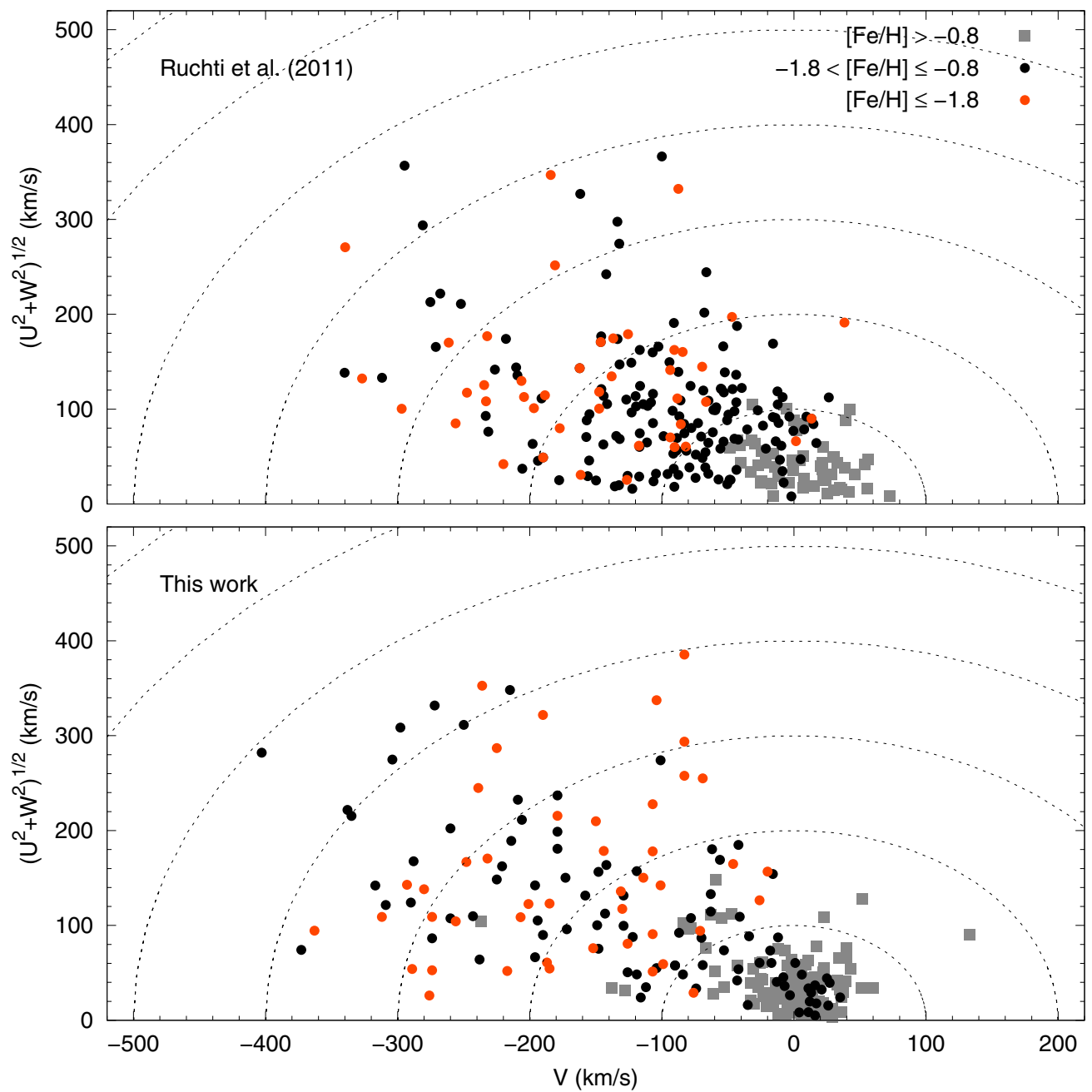

Figure 11. Toomre diagram of $\left(U^{2}+W^{2}\right)^{1 / 2}$ vs. $V$ for the stars with available $U V W$ velocity components, in three regimes of metallicity, for stars the sample of RAVE stars from Ruchti et al. (2011) (upper panel) and in the B\&M sample (lower panel). Note the presence of the intermediate-metallicity $(-1.8<[\mathrm{Fe} / \mathrm{H}] \leqslant-0.8)$ stars both inside and outside the region with low $\left(U^{2}+W^{2}\right)^{1 / 2}$ and high orbital rotation velocities $\left(\left(U^{2}+W^{2}\right)^{1 / 2} \lesssim 100 \mathrm{~km} \mathrm{~s}^{-1},-100<V<100 \mathrm{~km} \mathrm{~s}^{-1}\right)$. Stars with errors in any of the individual derived components of motion exceeding $50 \mathrm{~km} \mathrm{~s}^{-1}$ are excluded.

(A color version of this figure is available in the online journal.)

some overlap with the thin disk and MWTD is inevitable. Stars that are likely members of the disk system are identified by a " 1 " in the second digit of the INOUT parameter in Column 18 of Table 5. Stars with metallicities in the high range noted in this upper grouping of panels are identified by a " 1 " in the third digit of the INOUT parameter.

The middle grouping of panels are similar plots, but now for stars from each sample with intermediate metallicities, $-1.8<$ $[\mathrm{Fe} / \mathrm{H}] \leqslant-0.8$, which corresponds to an interval expected to have a significant contribution of MWTD stars (again with possible overlap from other stellar populations). Once more the top row of plots is the full sample of stars, and the bottom row is the subset in this interval that falls in the disk sample. Stars in the upper panel of this grouping would presumably include members of the "local halo" component proposed by Morrison et al. (2009). Unfortunately, neither the B\&M sample nor the Ruchti et al. sample contains a sufficient number of stars to explore this issue in further detail. So here we simply note the possibility of its presence. Stars with metallicities in the intermediate range noted in this grouping of panels are identified by a "2" in the third digit of the INOUT parameter in Column 18 of Table 5.
The lower grouping of panels compares the distribution of $V_{\phi}$ for the two metallicity intervals considered previously, but only for the stars in the disk samples. We employ a two-sample Kolmogorov-Smirnov (K-S) test to check if the subset of disk stars chosen from the B\&M sample and Ruchti et al. samples in the high- and intermediate-metallicity intervals are consistent with selection from the same parent population. Indeed, this test cannot reject the common-parent null hypothesis for either metallicity interval, in spite of the fact that the samples were clearly chosen in different ways. In contrast, it is unsurprising that the same K-S test applied between the disk stars in the different metallicity intervals for each of the B\&M and Ruchti et al. samples clearly discriminates between their distributions of $V_{\phi}$.

The black vertical lines mark the location of the $\left\langle V_{\phi}\right\rangle$ for each disk subsample. The means and dispersions of the stars we would associate with the canonical thick-disk population for the B\&M sample (the metallicity interval $-0.8<[\mathrm{Fe} / \mathrm{H}] \leqslant-0.5$ ) are $\left\langle V_{\phi}\right\rangle=216 \mathrm{~km} \mathrm{~s}^{-1}, \sigma_{V_{\phi}}=37 \mathrm{~km} \mathrm{~s}^{-1}$, while those for the Ruchti et al. sample are $\left\langle V_{\phi}\right\rangle=229 \mathrm{~km} \mathrm{~s}^{-1}, \sigma_{V_{\phi}}=30 \mathrm{~km} \mathrm{~s}^{-1}$. Note that the values we obtain for the mean rotational velocity of the thick-disk population from this crude analysis are 

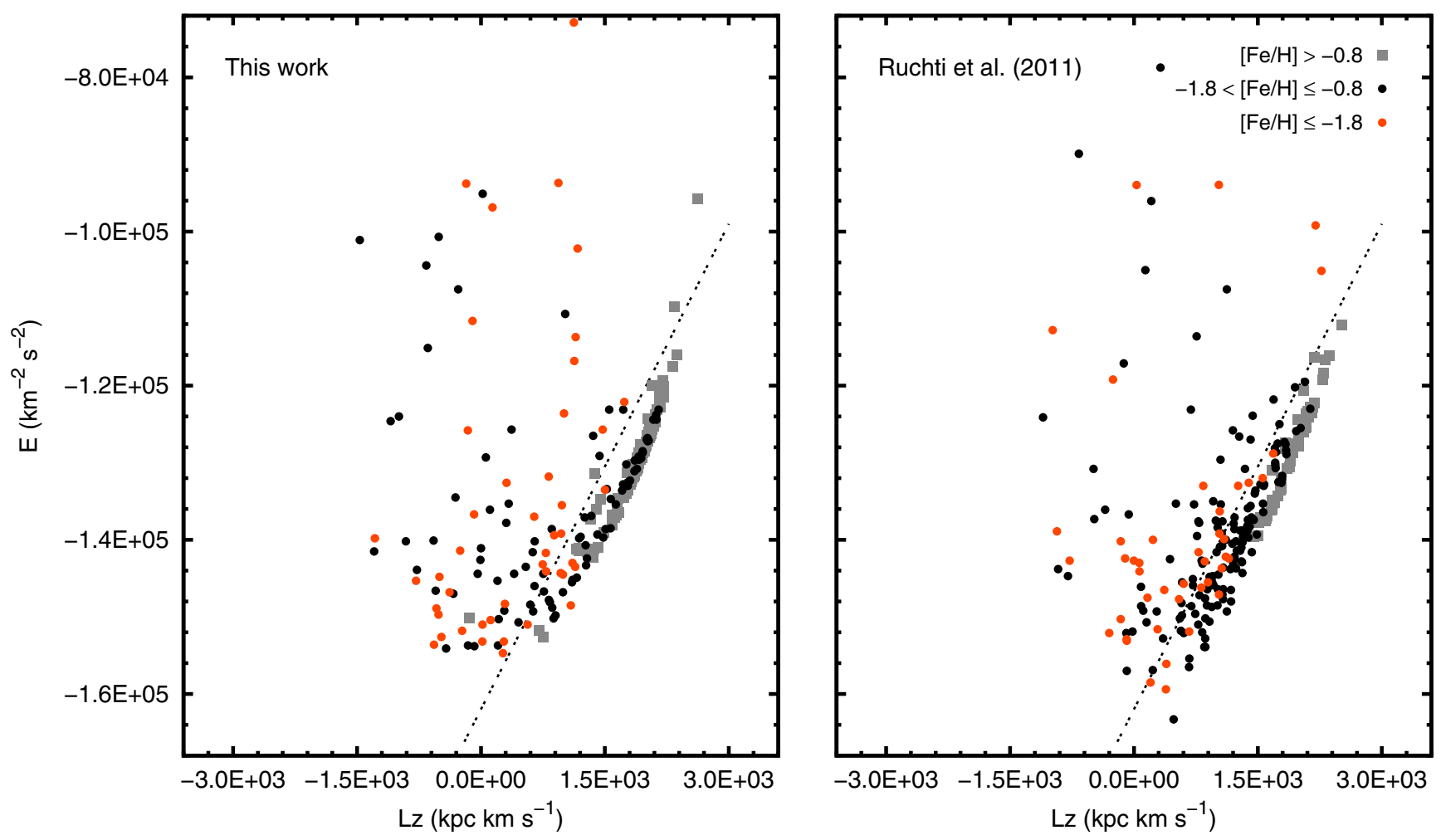

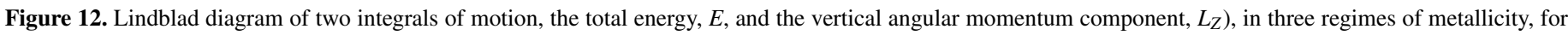

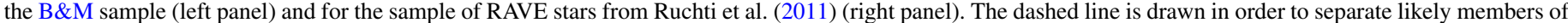

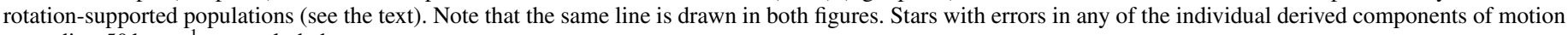
exceeding $50 \mathrm{~km} \mathrm{~s}^{-1}$ are excluded.

(A color version of this figure is available in the online journal.)

somewhat higher, and the velocity dispersions somewhat lower, than those reported by Carollo et al. (2010) $\left(\left\langle V_{\phi}\right\rangle=182 \mathrm{~km} \mathrm{~s}^{-1}\right.$, $\left.\sigma_{V_{\phi}}=51 \mathrm{~km} \mathrm{~s}^{-1}\right)$. It stands to reason that contamination from stars of the thin-disk population in this interval may be at least in part responsible for this result. However, it is also worth noting that Chiba \& Beers (2000), and other authors since, have reported that the asymmetric drift of the thick disk exhibits a strong gradient with distance above the Galactic plane, on the order of $\Delta\left\langle V_{\phi}\right\rangle / \Delta|Z|=36 \mathrm{~km} \mathrm{~s}^{-1} \mathrm{kpc}^{-1}$, according to Carollo et al. The B\&M sample and the Ruchti et al. disk sample are both located very close to the plane, and hence would be expected to be in more rapid rotation. In the case of stars we would associate with a MWTD population (the metallicity interval $-1.8<$ $[\mathrm{Fe} / \mathrm{H}] \leqslant-0.8)$, we obtain $\left\langle V_{\phi}\right\rangle=181 \mathrm{~km} \mathrm{~s}^{-1}, \sigma_{V_{\phi}}=$ $53 \mathrm{~km} \mathrm{~s}^{-1}$, while those for the Ruchti et al. sample are $\left\langle V_{\phi}\right\rangle=$ $166 \mathrm{~km} \mathrm{~s}^{-1}, \sigma_{V_{\phi}}=47 \mathrm{~km} \mathrm{~s}^{-1}$. The rotational velocity of the MWTD in the present analysis is again somewhat higher than reported by Carollo et al., who obtained a mean rotational velocity of this component of $\left\langle V_{\phi}\right\rangle \sim 100-150 \mathrm{~km} \mathrm{~s}^{-1}$, with a dispersion in the range $35-45 \mathrm{~km} \mathrm{~s}^{-1}$, which is a little lower than our derived value. Contamination of the B\&M and Ruchti et al. samples from metal-poor stars of the inner-halo population is possibly responsible for this result. The difference in the mean rotational velocity might also be accounted for if, as speculated by Carollo et al., the MWTD also exhibits a gradient in its rotational velocity with distance from the plane; future tests with larger samples should prove illuminating.

\subsection{Distribution of $[\mathrm{C} / \mathrm{Fe}]$ for the $B \& M$ Sample}

Figure 14 shows the distribution of carbonicity, $[\mathrm{C} / \mathrm{Fe}]$, as a function of $[\mathrm{Fe} / \mathrm{H}]$, for the stars in the B\&M sample.
The general increase in the level of $[\mathrm{C} / \mathrm{Fe}]$ with decreasing $[\mathrm{Fe} / \mathrm{H}]$, as has been seen in numerous previous samples, is evident. There are 12 stars in this sample which are classified as CEMP stars (and tagged as such in Table 3). Their frequency appears to increase with declining $[\mathrm{Fe} / \mathrm{H}]$, again as noted in previous samples. Based on their metallicities, we expect that the majority of these stars will be classified as CEMP-s, rather than CEMP-no, once high-resolution spectroscopy has been carried out.

\section{DISCUSSION AND CONCLUSIONS}

As noted in the Introduction, a large number of recent papers on the nature, origin, and evolution of the disk (and halo) system of the Milky Way have raised new and important questions concerning issues that were once considered "solved." In this paper, we have sought to explore what can be learned from a modern analysis of a pioneering data set, the "weak-metal" sample of Bidelman \& MacConnell (1973) studied by Norris et al. (1985), Paper I of this series. Many of the ideas and insights from Paper I have spawned research directions that have not yet, even today, been completely explored to their conclusion. Here, we have focused on resolving one of the most important claims from that paper, the suggested existence for what has come to be known as the MWTD. As we have shown, the worries raised by a number of authors, subsequent to the publication of Paper I, were indeed valid. The faulty calibration of a photometric DDObased metallicity determination led to the production of a false signature in the analysis of Paper I, which may (or may not) have unduly influenced claims for the existence of a MWTD.

The combination of high-quality, high-S/N mediumresolution spectroscopic data with a set of tools capable of 

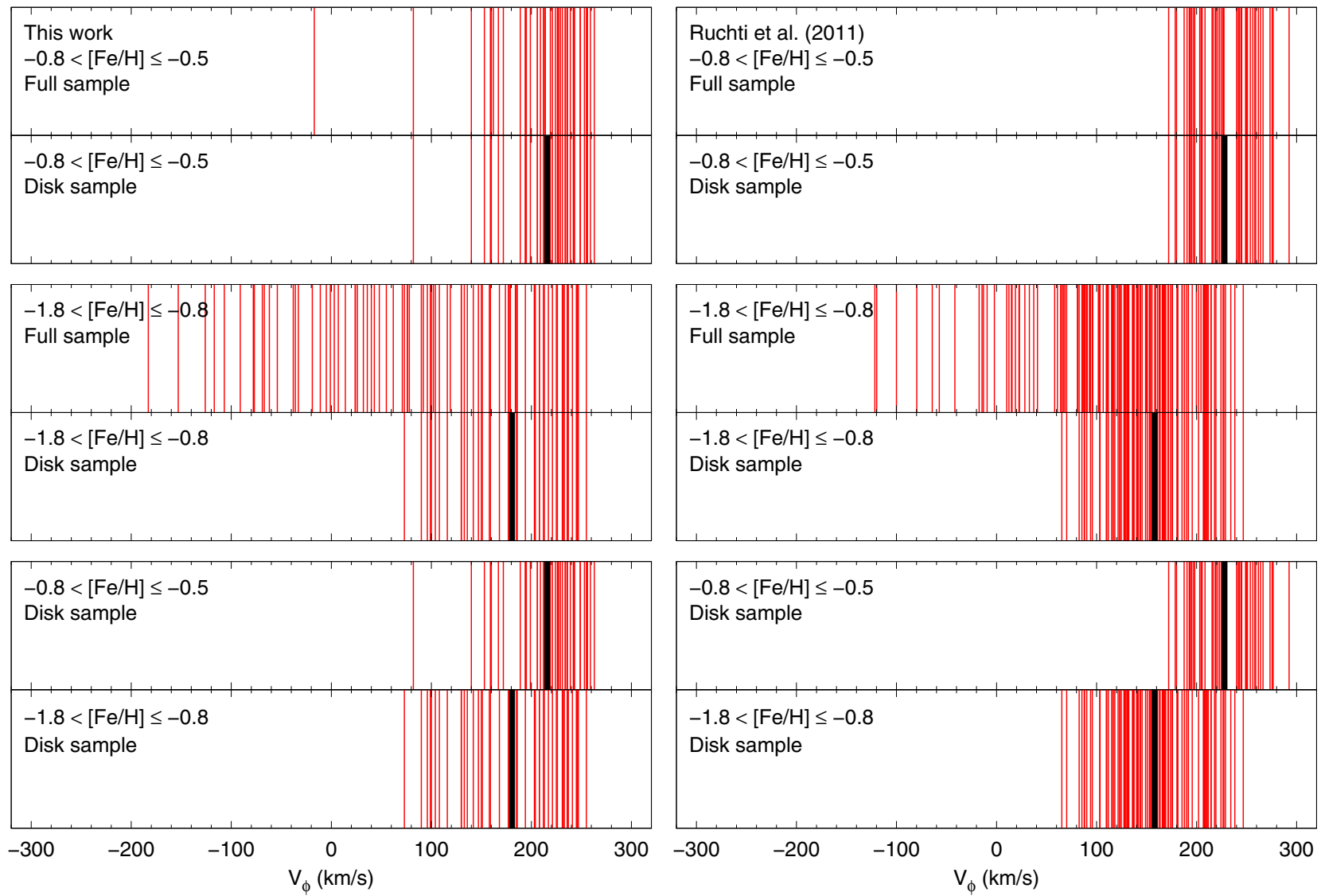

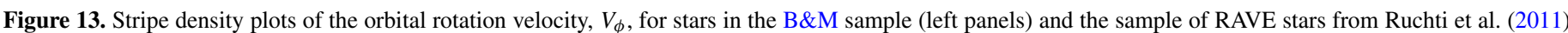

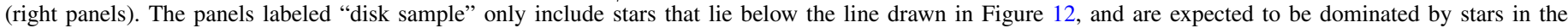

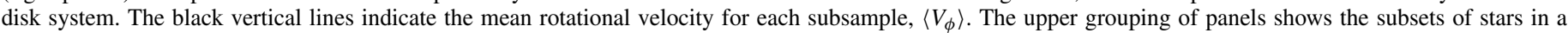

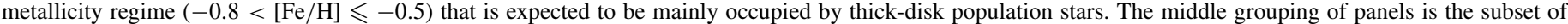

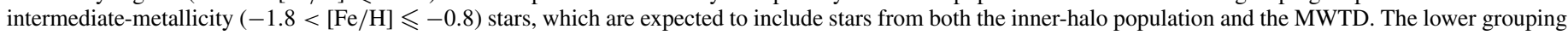

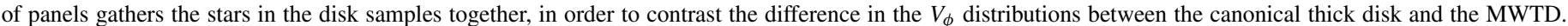
Stars with errors in any of the individual derived components of motion exceeding $50 \mathrm{~km} \mathrm{~s}^{-1}$ are excluded.

(A color version of this figure is available in the online journal.)

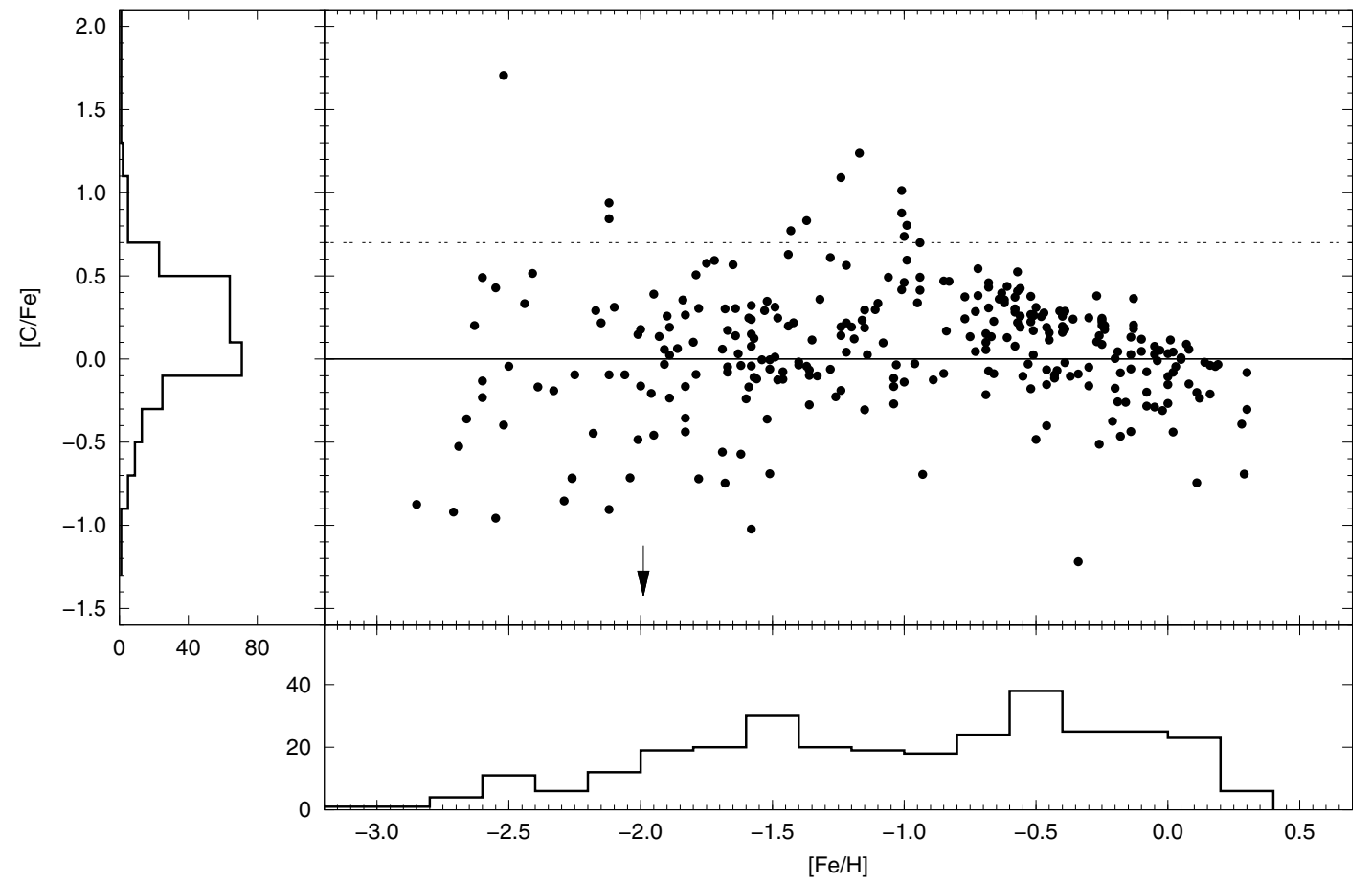

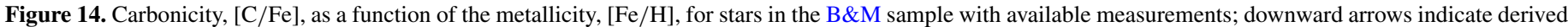

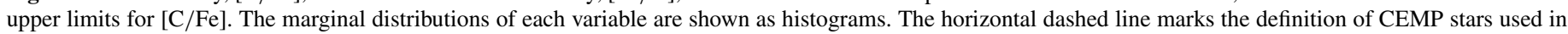
this work, $[\mathrm{C} / \mathrm{Fe}] \geqslant+0.7$. 
producing accurate, and well-tested, estimates of the atmospheric parameters (and carbonicity, $[\mathrm{C} / \mathrm{Fe}]$ ) for the same sample of stars as analyzed in Paper I, has allowed us to carry out a new (and expanded) consideration of the presence of stars that might be associated with a MWTD. We also have the advantage that we could make use of much-improved RVs and proper motions than were available to the previous study, adding substantially to the size of the data set suitable for kinematic study, while simultaneously improving the quality of the derived kinematics.

We conclude that the data set from Paper I does indeed comprise stars that can be associated with a MWTD. A comparison of these data with a similar-size sample from the RAVE survey (and with atmospheric parameters determined from a completely different set of techniques) yields essentially the same conclusion. We note, however, as pointed out by an anonymous referee, that both samples of stars we have considered are impacted by metallicity-selection biases (and in the case of the Ruchti et al. sample, by kinematic-selection bias as well). Thus, the relative numbers of stars present in any given metallicity interval should clearly not be taken as representative of the underlying parent population. We have also not addressed in this paper whether or not a MWTD is indeed best considered a separate component from the rest of the disk system, or whether it is somehow causally linked to the same formation processes (themselves still actively debated) involved with the origin of the thick (and even thin) disks. We leave these issues to future work.

We thank an anonymous referee for remarks which served to improve our manuscript. T.C.B. acknowledges partial support for this work from grants PHY 08-22648; Physics Frontier Center/Joint Institute for Nuclear Astrophysics (JINA), and PHY 14-30152; Physics Frontier Center/JINA Center for the Evolution of the Elements (JINA-CEE) awarded by the US National Science Foundation. J.E.N. acknowledges support from Australian Research Council grants DP0663562 and DP0984924. V.M.P. acknowledges support from the Gemini Observatory. S.R. acknowledges partial support from FAPESP, CNPq, and Capes. T.M. acknowledges partial support from the European Union FP7 program through ERC grant 320360. Y.S.L. is a Tombaugh Fellow. D.C. is an Australian Research Council Super Science Fellow.

\section{REFERENCES}

Adibekyan, V. Z., Figueira, P., Santos, N. C., et al. 2013, A\&A, 554, A44 Allende Prieto, C., Fernandez-Alvar, E., Schlesinger, K., et al. 2014, A\&A, 568,

Allende Prieto, C., Sivarani, T., Beers, T. C., et al. 2008, AJ, 136, 2070

An, D., Beers, T. C., Johnson, J. A., et al. 2013, ApJ, 763, 65

Anthony-Twarog, B. J., \& Twarog, B. A. 1994, AJ, 107, 1577

Aoki, W., Beers, T. C., Lee, Y. S., et al. 2013, AJ, 145, 13

Arifyanto, M. I., Fuchs, B., Jahreiß, H., \& Wielen, R. 2005, A\&A, 433, 911

Beers, T. C., Carollo, D., Ivezić, Ž., et al. 2012, ApJ, 746, 34

Beers, T. C., Chiba, M., Yoshii, Y., et al. 2000, AJ, 119, 2866

Beers, T. C., Drilling, J. S., Rossi, S., et al. 2002, AJ, 124, 931

Beers, T. C., Preston, G. W., \& Shectman, S. A. 1985, AJ, 90, 2089

Beers, T. C., Preston, G. W., \& Shectman, S. A. 1992, AJ, 103, 1987

Beers, T. C., Rossi, S., Norris, J. E., Ryan, S. G., \& Shefler, T. 1999, AJ, 117,981

Beers, T. C., \& Sommer-Larsen, J. 1995, ApJS, 96, 175

Bensby, T., Feltzing, S., \& Oey, S. 2014, A\&A, 562, 71
Bidelman, W. P., \& MacConnell, D. J. 1973, AJ, 78, 687

Boeche, C., Chiappini, C., Minchev, I., et al. 2013a, A\&A, 553, 19

Boeche, C., Siebert, A., Piffl, T., et al. 2013b, A\&A, 559, 59

Bovy, J., Rix, H.-W., \& Hogg, D. W. 2012a, ApJ, 751, 131

Bovy, J., Rix, H.-W., Hogg, D. W., et al. 2012b, ApJ, 755, 115

Bovy, J., Rix, H.-W., Liu, C., et al. 2012c, ApJ, 753, 148

Bovy, J., Rix, H.-W., Liu, C., et al. 2012d, ApJ, 753, 148

Carney, B. W., Latham, D. W., Laird, J. B., \& Aguilar, L. A. 1994, AJ, 107, 2240

Carollo, D., Beers, T. C., Bovy, J., et al. 2012, ApJ, 744, 195

Carollo, D., Beers, T. C., Chiba, M., et al. 2010, ApJ, 712, 692

Carollo, D., Beers, T. C., Lee, Y. S., et al. 2007, Natur, 450, 1020

Carollo, D., Freeman, K., Beers, T. C., et al. 2014, ApJ, 788, 180

Carrell, K., Chen, Y., \& Zhao, G. 2012, AJ, 144, 185

Cayrel de Strobel, G., Soubiran, C., \& Ralite, N. 2001, A\&A, 373, 159

Cheng, J. Y., Rockosi, C. M., Morrison, H. L., et al. 2012a, ApJ, 752, 51

Cheng, J. Y., Rockosi, C. M., Morrison, H. L., et al. 2012b, ApJ, 746, 149

Chiba, M., \& Beers, T. C. 2000, AJ, 119, 2843

Chiba, M., \& Yoshii, Y. 1998, AJ, 115, 168

Drilling, J. S., \& Bergeron, L. E. 1995, PASP, 107, 846

Eggen, O. J., Lynden-Bell, D., \& Sandage, A. R. 1962, ApJ, 136, 748

Eisenstein, D. J., Weinberg, D. H., Agol, E., et al. 2011, AJ, 142, 72

ESA 1997, yCat, 1239, 0

Frebel, A. 2010, AN, 331, 474

Ghez, A. M., Salim, S., Weinberg, N. N., et al. 2008, ApJ, 689, 1044

Gilmore, G. 1984, MNRAS, 207, 223

Gilmore, G., Wyse, R. F. G., \& Norris, J. E. 2002, ApJL, 574, L39

Haywood, M., Di Matteo, P., Lehnert, M. D., Katz, D., \& Gómez, A. 2013, A\&A, 560, A109

Høg, E., Fabricius, C., Makarov, V. V., et al. 2000, A\&A, 355, L27

Hog, E., Kuzmin, A., Bastian, U., et al. 1998, A\&A, 335, L65

Jayaraman, A., Gilmore, G., Wyse, R. F. G., Norris, J. E., \& Belokurov, V. 2013, MNRAS, 431, 930

Kerr, F. J., \& Lynden-Bell, D. 1986, MNRAS, 221, 1023

Koposov, S. E., Yoo, J., Rix, H.-W., et al. 2009, ApJ, 696, 2179

Kordopatis, G., Gilmore, G., Wyse, R. F. G., et al. 2013a, MNRAS, 436, 3231

Kordopatis, G., Hill, V., Irwin, M., et al. 2013b, A\&A, 555, 12

Kordopatis, G., Recio-Blanco, A., de Laverny, P., et al. 2011, A\&A, 535, A107

Layden, A. C. 1995, AJ, 110, 2288

Lee, Y. S., Beers, T. C., Allende Prieto, C., et al. 2011, AJ, 141, 90

Lee, Y. S., Beers, T. C., Masseron, T., et al. 2013, AJ, 146, 132

Lee, Y. S., Beers, T. C., Sivarani, T., et al. 2008a, AJ, 136, 2022

Lee, Y. S., Beers, T. C., Sivarani, T., et al. 2008b, AJ, 136, 2050

Majewski, S. R., Wilson, J. C., Hearty, F., Schiavon, R. R., \& Skrutskie, M. F. 2010, in IAU Symp. 265, ed. K. Cunha, M. Spite, \& B. Barbuy (Cambridge: Cambrdge Univ. Press), 480

Martin, J. C., \& Morrison, H. L. 1998, AJ, 116, 1724

Mihalas, D., \& Binney, J. 1981, Galactic Astronomy: Structure and kinematics (2nd ed.; San Francisco, CA: Freeman)

Minchev, I., Chiappini, C., Martig, M., et al. 2014, ApJL, 781, L20

Morrison, H. L., Flynn, C., \& Freeman, K. C. 1990, AJ, 100, 1191

Morrison, H. L., Helmi, A., Sun, J., et al. 2009, ApJ, 694, 130

Norris, J. E., Bessell, M. S., \& Pickles, A. J. 1985, ApJS, 58, 463 (Paper I)

Norris, J. E., Ryan, S. G., \& Beers, T. C. 1999, ApJS, 123, 639

Pasetto, S., Grebel, E. K., Zwitter, T., et al. 2012, A\&A, 547, 70

Ruchti, G. R., Fulbright, J. P., Wyse, R. F. G., et al. 2010, ApJL, 721, L92

Ruchti, G. R., Fulbright, J. P., Wyse, R. F. G., et al. 2011, ApJ, 737, 9

Ryan, S. G., \& Lambert, D. L. 1995, AJ, 109, 2068

Schlegel, D. J., Finkbeiner, D. P., \& Davis, M. 1998, ApJ, 500, 525

Schönrich, R., Asplund, M., \& Casagrande, L. 2011, MNRAS, 415, 3807

Shortridge, K. 1993, in ASP Conf. Ser. 52, Astronomical Data Analysis Software and Systems II, ed. R. J. Hanisch, R. J. V. Brissenden, \& J. Barnes (San Francisco, CA: ASP), 219

Skrutskie, M. F., Cutri, R. M., Stiening, R., et al. 2006, AJ, 131, 1163

Smolinski, J. P., Lee, Y. S., Beers, T. C., et al. 2011, AJ, 141, 89

Soubiran, C., Le Campion, J.-F., Cayrel de Strobel, G., \& Caillo, A. 2010, A\&A, 515, A111

Steinmetz, M., Zwitter, T., Siebert, A., et al. 2006, AJ, 132, 1645

Suda, T., Katsuta, Y., Yamada, S., et al. 2008, PASJ, 60, 1159

van Leeuwen, F. 2007, A\&A, 474, 653

Villalobos, Á., \& Helmi, A. 2009, MNRAS, 399, 166

Yanny, B., Rockosi, C., Newberg, H. J., et al. 2009, AJ, 137, 4377

York, D. G., Adelman, J., Anderson, J. E., Jr., et al. 2000, AJ, 120, 1579 